\title{
A fourth-order compact time-splitting Fourier pseudospectral method for the Dirac equation
}

\author{
Weizhu Bao · Jia Yin
}

Received: date / Accepted: date

\begin{abstract}
We propose a new fourth-order compact time-splitting $\left(S_{4 \mathrm{c}}\right)$ Fourier pseudospectral method for the Dirac equation by splitting the Dirac equation into two parts together with using the double commutator between them to integrate the Dirac equation at each time interval. The method is explicit, fourth-order in time and spectral order in space. It is unconditional stable and conserves the total probability in the discretized level. It is called a compact time-splitting method since, at each time step, the number of sub-steps in $S_{4 \mathrm{c}}$ is much less than those of the standard fourth-order splitting method and the fourth-order partitioned Runge-Kutta splitting method. Another advantage of $S_{4 \mathrm{c}}$ is that it avoids to use negative time steps in integrating sub-problems at each time interval. Comparison between $S_{4 \mathrm{c}}$ and many other existing time-splitting methods for the Dirac equation are carried out in terms of accuracy and efficiency as well as long time behavior. Numerical results demonstrate the advantage in terms of efficiency and accuracy of the proposed $S_{4 \mathrm{c}}$. Finally we report the spatial/temporal resolutions of $S_{4 \mathrm{c}}$ for the Dirac equation in different parameter regimes including the nonrelativistic limit regime, the semiclassical limit regime, and the simultaneously nonrelativisic and massless limit regime.
\end{abstract}

This work was partially supported by the Ministry of Education of Singapore grant R-146-000-223-112 (MOE2015-T2-2-146).

Weizhu Bao

Department of Mathematics, National University of Singapore, Singapore 119076, Singapore

E-mail: matbaowz@nus.edu.sg

URL: http://www.math.nus.edu.sg/־bao/

Jia Yin (Corresponding author)

NUS Graduate School for Integrative Sciences and Engineering (NGS), National University of Singapore, Singapore 117456, Singapore

E-mail: e0005518@u.nus.edu 
Keywords Dirac equation - fourth-order compact time-splitting - double commutator · probability conservation $\cdot$ nonrelativistic limit regime $\cdot$ semiclassical limit regime

\section{Introduction}

The Dirac equation was proposed by British physicist Paul Dirac in 1928 in order to integrate special relativity with quantum mechanics [29]. It successfully solved the problem that the probability density could be negative in the Klein-Gordon equation proposed by Oskar Klein and Walter Gordon in 1926 [28]. The Dirac equation describes the motion of relativistic spin$1 / 2$ massive particles, such as electrons and quarks. It fully explained the hydrogen spectrum and predicted the existence of antimatter. Recently, the Dirac equation has been extensively adopted to investigate theoretically the structures and/or dynamical properties of graphene and graphite as well as other two-dimensional (2D) materials [1,33,49,48], and to study the relativistic effects in molecules in super intense lasers, e.g., attosecond lasers [17,36].

Consider the Dirac equation with electromagnetic potentials in three spatial dimensions (3D) $29,30,31,60$

$$
i \hbar \partial_{t} \Psi=\left(-i c \hbar \sum_{j=1}^{3} \alpha_{j} \partial_{j}+m c^{2} \beta\right) \Psi+e\left(V(\mathbf{x}) I_{4}-\sum_{j=1}^{3} A_{j}(\mathbf{x}) \alpha_{j}\right) \Psi, \quad \mathbf{x} \in \mathbb{R}^{3},
$$

where $t$ is time, $\mathbf{x}=\left(x_{1}, x_{2}, x_{3}\right)^{T}$ (or $\left.\mathbf{x}=(x, y, z)^{T}\right)$ is the spatial coordinate, $\Psi:=\Psi(t, \mathbf{x})=$ $\left(\psi_{1}(t, \mathbf{x}), \psi_{2}(t, \mathbf{x}), \psi_{3}(t, \mathbf{x}), \psi_{4}(t, \mathbf{x})\right)^{T} \in \mathbb{C}^{4}$ is the complex-valued spinor wave function, and $\partial_{j}$ represents $\partial_{x_{j}}$ for $j=1,2,3$. The constants used in the equation are: $i=\sqrt{-1}, \hbar$ is the Planck constant, $m$ is the mass, $c$ is the speed of light and $e$ is the unit charge. In addition, $V:=V(\mathbf{x})$ is the time-independent electric potential and $\mathbf{A}:=\mathbf{A}(\mathbf{x})=\left(A_{1}(\mathbf{x}), A_{2}(\mathbf{x}), A_{3}(\mathbf{x})\right)^{T}$ stands for the time-independent magnetic potential, which are all real-valued given functions. Finally, the $4 \times 4$ matrices $\beta$ and $\alpha_{j}(j=1,2,3)$ are the Dirac representation matrices of the four-dimensional Clifford algebra, which are given as

$$
\beta=\left(\begin{array}{cc}
I_{2} & \mathbf{0} \\
\mathbf{0} & -I_{2}
\end{array}\right), \quad \alpha_{j}=\left(\begin{array}{cc}
\mathbf{0} & \sigma_{j} \\
\sigma_{j} & \mathbf{0}
\end{array}\right), \quad j=1,2,3,
$$

where $I_{n}$ is the $n \times n$ identity matrix and $\sigma_{j}(j=1,2,3)$ are the Pauli matrices defined as:

$$
\sigma_{1}=\left(\begin{array}{ll}
0 & 1 \\
1 & 0
\end{array}\right), \quad \sigma_{2}=\left(\begin{array}{cc}
0 & -i \\
i & 0
\end{array}\right), \quad \sigma_{3}=\left(\begin{array}{cc}
1 & 0 \\
0 & -1
\end{array}\right)
$$

In order to nondimensionalize the Dirac equation (1.1), we take

$$
\tilde{\mathbf{x}}=\frac{\mathbf{x}}{x_{s}}, \quad \tilde{t}=\frac{t}{t_{s}}, \quad \tilde{V}=\frac{V}{A_{s}}, \quad \tilde{\mathbf{A}}=\frac{\mathbf{A}}{A_{s}}, \quad \tilde{\Psi}(\tilde{t}, \tilde{\mathbf{x}})=\frac{\Psi(t, \mathbf{x})}{\psi_{s}},
$$


where $x_{s}, t_{s}$ and $m_{s}$ are length unit, time unit and mass unit, respectively, to be taken for the nondimensionalization of the Dirac equation (1.1). Plugging (1.4) into (1.1) and taking

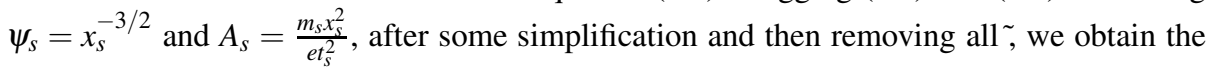
dimensionless Dirac equation in 3D

$$
i \delta \partial_{t} \Psi=\left(-i \frac{\delta}{\varepsilon} \sum_{j=1}^{3} \alpha_{j} \partial_{j}+\frac{v}{\varepsilon^{2}} \beta\right) \Psi+\left(V(\mathbf{x}) I_{4}-\sum_{j=1}^{3} A_{j}(\mathbf{x}) \alpha_{j}\right) \Psi, \quad \mathbf{x} \in \mathbb{R}^{3},
$$

where the three dimensionless parameters $0<\varepsilon, \delta, v \leq 1$ are given as

$$
\varepsilon=\frac{x_{s}}{t_{s} c}=\frac{v_{s}}{c}, \quad \delta=\frac{\hbar t_{s}}{m_{s} x_{s}^{2}}, \quad v=\frac{m}{m_{s}}
$$

with $v_{s}=x_{s} / t_{s}$ the velocity unit for nondimensionalization. In fact, here $\varepsilon$ represents the ratio between the wave velocity and the speed of light, i.e. it is inversely proportional to the speed of light, $\delta$ stands for the scaled Planck constant and $v$ is the ratio between the mass of the particle and the mass unit taken for the nondimensionalization.

As discussed in [9], under proper assumption on the electromagnetic potentials $V(\mathbf{x})$ and $\mathbf{A}(\mathbf{x})$, the Dirac equation (1.5) in 3D could be reduced to two dimensions (2D) and one dimension (1D). Specifically, the Dirac equation in 2D has been widely applied to model the electron structure and dynamical properties of graphene and other 2D materials as they share the same dispersion relation on certain points called Dirac points [33,34,35,48]. In fact, the Dirac equation (1.5) in 3D and its dimension reduction in 2D and $1 \mathrm{D}$ can be formulated in a unified way in $d$-dimensions $(d=1,2,3)$ as

$$
i \delta \partial_{t} \Psi=\left(-i \frac{\delta}{\varepsilon} \sum_{j=1}^{d} \alpha_{j} \partial_{j}+\frac{v}{\varepsilon^{2}} \beta\right) \Psi+\left(V(\mathbf{x}) I_{4}-\sum_{j=1}^{d} A_{j}(\mathbf{x}) \alpha_{j}\right) \Psi, \quad \mathbf{x} \in \mathbb{R}^{d},
$$

where $\mathbf{x}=\left(x_{1}, x_{2}\right)^{T}$ (or $\left.\mathbf{x}=(x, y)^{T}\right)$ in $2 \mathrm{D}$ and $\mathbf{x}=x_{1}$ (or $\left.\mathbf{x}=x\right)$ in 1D. To study the dynamics of the Dirac equation (1.7), the initial condition is usually taken as

$$
\Psi(t=0, \mathbf{x})=\Psi_{0}(\mathbf{x}), \quad \mathbf{x} \in \mathbb{R}^{d} .
$$

The Dirac equation (1.7) with (1.8) is dispersive, time-symmetric, and it conserves the total probability [9]

$$
\|\Psi(t, \cdot)\|^{2}:=\int_{\mathbb{R}^{d}}|\Psi(t, \mathbf{x})|^{2} d \mathbf{x}=\int_{\mathbb{R}^{d}} \sum_{j=1}^{4}\left|\psi_{j}(t, \mathbf{x})\right|^{2} d \mathbf{x} \equiv\|\Psi(0, \cdot)\|^{2}=\left\|\Psi_{0}\right\|^{2}, \quad t \geq 0
$$


and the energy [9]

$$
\begin{aligned}
E(\Psi(t, \cdot)) & :=\int_{\mathbb{R}^{d}}\left(-i \frac{\delta}{\varepsilon} \sum_{j=1}^{d} \Psi^{*} \alpha_{j} \partial_{j} \Psi+\frac{v}{\varepsilon^{2}} \Psi^{*} \beta \Psi+V(\mathbf{x})|\Psi|^{2}-\sum_{j=1}^{d} A_{j}(\mathbf{x}) \Psi^{*} \alpha_{j} \Psi\right) d \mathbf{x} \\
& \equiv E\left(\Psi_{0}\right), \quad t \geq 0
\end{aligned}
$$

where $\Psi^{*}=\bar{\Psi}^{T}$ with $\bar{f}$ denoting the complex conjugate of $f$.

Introduce the total probability density $\rho:=\rho(t, \mathbf{x})$ as

$$
\rho(t, \mathbf{x})=\sum_{j=1}^{4} \rho_{j}(t, \mathbf{x})=\Psi(t, \mathbf{x})^{*} \Psi(t, \mathbf{x}), \quad \mathbf{x} \in \mathbb{R}^{d},
$$

where the probability density $\rho_{j}:=\rho_{j}(t, \mathbf{x})$ of the $j$-th $(j=1,2,3,4)$ component is defined as

$$
\rho_{j}(t, \mathbf{x})=\left|\psi_{j}(t, \mathbf{x})\right|^{2}, \quad \mathbf{x} \in \mathbb{R}^{d},
$$

and the current density $\left.\mathbf{J}(t, \mathbf{x})=\left(J_{1}(t, \mathbf{x}), \ldots, J_{d}(t, \mathbf{x})\right)\right)^{T}$ as

$$
J_{l}(t, \mathbf{x})=\frac{1}{\varepsilon} \Psi(t, \mathbf{x})^{*} \alpha_{l} \Psi(t, \mathbf{x}), \quad l=1, \ldots, d,
$$

then the following conservation law can be obtained from the Dirac equation (1.7) [9]

$$
\partial_{t} \rho(t, \mathbf{x})+\nabla \cdot \mathbf{J}(t, \mathbf{x})=0, \quad \mathbf{x} \in \mathbb{R}^{d}, \quad t \geq 0 .
$$

If the electric potential $V$ is perturbed by a real constant $V^{0}$, i.e., $V \rightarrow V+V^{0}$, then the solution $\Psi(t, \mathbf{x}) \rightarrow e^{-i \frac{V^{0} t}{\delta}} \Psi(t, \mathbf{x})$, which implies that the probability density of each component $\rho_{j}(j=1,2,3,4)$ and the total probability density $\rho$ are all unchanged. In addition, when $d=1$, if the magnetic potential $A_{1}$ is perturbed by a real constant $A_{1}^{0}$, i.e., $A_{1} \rightarrow A_{1}+A_{1}^{0}$, then the solution $\Psi(t, \mathbf{x}) \rightarrow e^{i \frac{A_{1}^{0} t}{\delta}} \alpha_{1} \Psi(t, \mathbf{x})$, which implies that only the total probability density $\rho$ is unchanged; however, this property is unfortunately not valid in 2D and 3D. Furthermore, if the external electromagnetic potentials are all real constants, i.e. $V(\mathbf{x}) \equiv V^{0}$ and $A_{j}(\mathbf{x}) \equiv A_{j}^{0}(j=1, \ldots, d)$ with $\mathbf{A}^{0}=\left(A_{1}^{0}, \ldots, A_{d}^{0}\right)^{T}$, the Dirac equation (1.7) admits the plane wave solution $\Psi(t, \mathbf{x})=\mathbf{B} e^{i\left(\mathbf{k} \cdot \mathbf{x}-\frac{\omega}{\delta} t\right)}$ with $\omega$ the time frequency, $\mathbf{B} \in \mathbb{R}^{4}$ the amplitude vector and $\mathbf{k}=\left(k_{1}, \ldots, k_{d}\right)^{T} \in \mathbb{R}^{d}$ the spatial wave number, which satisfies the following eigenvalue problem

$$
\omega \mathbf{B}=\left(\sum_{j=1}^{d}\left(\frac{\delta k_{j}}{\varepsilon}-A_{j}^{0}\right) \alpha_{j}+\frac{v}{\varepsilon^{2}} \beta+V^{0} I_{4}\right) \mathbf{B} .
$$

Solving the above equation, we can get the dispersion relation of the Dirac equation (1.7)

$$
\omega:=\omega(\mathbf{k})=V^{0} \pm \frac{1}{\varepsilon^{2}} \sqrt{v^{2}+\varepsilon^{2}\left|\delta \mathbf{k}-\varepsilon \mathbf{A}^{0}\right|^{2}}, \quad \mathbf{k} \in \mathbb{R}^{d} .
$$


In $2 \mathrm{D}$ and $1 \mathrm{D}$, i.e. $d=2$ or 1 in (1.7), similar as those in [8], the Dirac equation (1.7) can be decoupled into two simplified PDEs with $\Phi:=\Phi(t, \mathbf{x})=\left(\phi_{1}(t, \mathbf{x}), \phi_{2}(t, \mathbf{x})\right)^{T} \in \mathbb{C}^{2}$ satisfying

$$
i \delta \partial_{t} \Phi=\left(-i \frac{\delta}{\varepsilon} \sum_{j=1}^{d} \sigma_{j} \partial_{j}+\frac{v}{\varepsilon^{2}} \sigma_{3}\right) \Phi+\left(V(\mathbf{x}) I_{2}-\sum_{j=1}^{d} A_{j}(\mathbf{x}) \sigma_{j}\right) \Phi, \quad \mathbf{x} \in \mathbb{R}^{d},
$$

where $\Phi=\left(\psi_{1}, \psi_{4}\right)^{T}$ (or $\Phi=\left(\psi_{2}, \psi_{3}\right)^{T}$ ). Again, to study the dynamics of the Dirac equation (1.17), the initial condition is usually taken as

$$
\Phi(t=0, \mathbf{x})=\Phi_{0}(\mathbf{x}), \quad \mathbf{x} \in \mathbb{R}^{d}
$$

Similarly, the Dirac equation (1.17) with 1.18 is dispersive, time-symmetric, and it conserves the total probability 9

$$
\begin{aligned}
\|\Phi(t, \cdot)\|^{2} & :=\int_{\mathbb{R}^{d}}|\Phi(t, \mathbf{x})|^{2} d \mathbf{x}=\int_{\mathbb{R}^{d}} \sum_{j=1}^{2}\left|\phi_{j}(t, \mathbf{x})\right|^{2} d \mathbf{x} \\
& \equiv\|\Phi(0, \cdot)\|^{2}=\left\|\Phi_{0}\right\|^{2}, \quad t \geq 0
\end{aligned}
$$

and the energy [9]

$$
\begin{aligned}
E(\Phi(t, \cdot)) & :=\int_{\mathbb{R}^{d}}\left(-i \frac{\delta}{\varepsilon} \sum_{j=1}^{d} \Phi^{*} \sigma_{j} \partial_{j} \Phi+\frac{v}{\varepsilon^{2}} \Phi^{*} \sigma_{3} \Phi+V(\mathbf{x})|\Phi|^{2}-\sum_{j=1}^{d} A_{j}(\mathbf{x}) \Phi^{*} \sigma_{j} \Phi\right) d \mathbf{x} \\
& \equiv E\left(\Phi_{0}\right), \quad t \geq 0 .
\end{aligned}
$$

Again, introduce the total probability density $\rho:=\rho(t, \mathbf{x})$ as

$$
\rho(t, \mathbf{x})=\sum_{j=1}^{2} \rho_{j}(t, \mathbf{x})=\Phi(t, \mathbf{x})^{*} \Phi(t, \mathbf{x}), \quad \mathbf{x} \in \mathbb{R}^{d}
$$

where the probability density $\rho_{j}:=\rho_{j}(t, \mathbf{x})$ of the $j$-th $(j=1,2)$ component is defined as

$$
\rho_{j}(t, \mathbf{x})=\left|\phi_{j}(t, \mathbf{x})\right|^{2}, \quad \mathbf{x} \in \mathbb{R}^{d},
$$

and the current density $\left.\mathbf{J}(t, \mathbf{x})=\left(J_{1}(t, \mathbf{x}), \ldots, J_{d}(t, \mathbf{x})\right)\right)^{T}$ as

$$
J_{l}(t, \mathbf{x})=\frac{1}{\varepsilon} \Phi(t, \mathbf{x})^{*} \sigma_{l} \Phi(t, \mathbf{x}), \quad l=1, \ldots, d,
$$

then the same conservation law (1.14) can be obtained from the Dirac equation (1.17) [9].

Similarly, if the electric potential $V$ is perturbed by a real constant $V^{0}$, i.e., $V \rightarrow V+$ $V^{0}$, then the solution $\Phi(t, \mathbf{x}) \rightarrow e^{-i \frac{V^{0} t}{\delta}} \Phi(t, \mathbf{x})$, which implies that the probability density of each component $\rho_{j}(j=1,2)$ and the total probability density $\rho$ are all unchanged. In addition, when $d=1$, if the magnetic potential $A_{1}$ is perturbed by a real constant $A_{1}^{0}$, i.e., 
$A_{1} \rightarrow A_{1}+A_{1}^{0}$, then the solution $\Phi(t, \mathbf{x}) \rightarrow e^{i \frac{A_{1}^{0} t}{\delta}} \sigma_{1} \Phi(t, \mathbf{x})$, which implies that only the total probability density $\rho$ is unchanged; however, this property is unfortunately not valid in $2 \mathrm{D}$. Furthermore, if the external electromagnetic potentials are all real constants, i.e. $V(\mathbf{x}) \equiv V^{0}$ and $A_{j}(\mathbf{x}) \equiv A_{j}^{0}(j=1, \ldots, d)$ with $\mathbf{A}^{0}=\left(A_{1}^{0}, \ldots, A_{d}^{0}\right)^{T}$, the Dirac equation (1.17) admits the plane wave solution $\Phi(t, \mathbf{x})=\mathbf{B} e^{i\left(\mathbf{k} \cdot \mathbf{x}-\frac{\omega}{\delta} t\right)}$ with $\omega$ the time frequency, $\mathbf{B} \in \mathbb{R}^{2}$ the amplitude vector and $\mathbf{k}=\left(k_{1}, \ldots, k_{d}\right)^{T} \in \mathbb{R}^{d}$ the spatial wave number, which satisfies the following eigenvalue problem

$$
\omega \mathbf{B}=\left(\sum_{j=1}^{d}\left(\frac{\delta k_{j}}{\varepsilon}-A_{j}^{0}\right) \sigma_{j}+\frac{v}{\varepsilon^{2}} \sigma_{3}+V^{0} I_{2}\right) \mathbf{B} .
$$

Solving the above equation, we can get the dispersion relation of the Dirac equation 1.17

$$
\omega:=\omega(\mathbf{k})=V^{0} \pm \frac{1}{\varepsilon^{2}} \sqrt{v^{2}+\varepsilon^{2}\left|\delta \mathbf{k}-\varepsilon \mathbf{A}^{0}\right|^{2}}, \quad \mathbf{k} \in \mathbb{R}^{d} .
$$

If one sets the mass unit $m_{s}=m$, length unit $x_{s}=\frac{\hbar}{m c}$, and time unit $t_{s}=\frac{x_{s}}{c}=\frac{\hbar}{m c^{2}}$, then $\varepsilon=\delta=v=1$, which corresponds to the classical (or standard) scaling. This choice of $x_{s}, m_{s}$ and $t_{s}$ is appropriate when the wave speed is at the same order of the speed of light. However, a different choice of $x_{s}, m_{s}$ and $t_{s}$ is more appropriate when the wave speed is much smaller than the speed of light. We remark here that the choice of $x_{s}, m_{s}$ and $t_{s}$ determines the observation scale of time evolution of the system and decides which phenomena can be resolved by discretization on specified spatial/temporal grids and which phenomena is visible by asymptotic analysis.

Different parameter regimes could be considered for the Dirac equation 1.7 (or 1.17), which are displayed in Fig. 1.1.

- Standard (or classical) regime, i.e. $\varepsilon=\delta=v=1\left(\Longleftrightarrow m_{s}=m, x_{s}=\frac{\hbar}{m c}\right.$, and $t_{s}=$ $\left.\frac{\hbar}{m c^{2}}\right)$, the wave speed is at the order of the speed of light. In this parameter regime, formally the dispersion relation (1.16) (or 1.25) suggests $\omega(\mathbf{k})=O(1)$ when $|\mathbf{k}|=$ $O(1)$ and thus the solution propagates waves with wavelength at $O(1)$ in space and time. In addition, if the initial data $\Psi_{0}=O(1)$ in 1.8$)$ (or $\Phi_{0}=O(1)$ in $(1.18)$ ), then the solution $\Psi=O(1)$ of (1.7) with 1.8) (or $\Phi=O(1)$ of (1.17) with 1.18), which implies that the probability density $\rho=O(1)$ in 1.11 (or 1.21 ), current density $\mathbf{J}=O(1)$ in (1.13) (or 1.23) and the energy $E(\Psi(t, \cdot))=O(1)$ in 1.10) (or $E(\Phi(t, \cdot))=O(1)$ in (1.20). There were extensive analytical and numerical studies for the Dirac equation (1.7) (or (1.17) ) with $\varepsilon=\delta=v=1$ in the literatures. For the existence and multiplicity of bound states and/or standing wave solutions, we refer to [26, 27, 32, 40, 41, 52] and references therein. In this parameter regime, for the numerical part, many efficient and accurate numerical methods have been proposed and analyzed [3], such as the finite difference time domain (FDTD) methods [4,50], time-splitting Fourier pseudospectral 


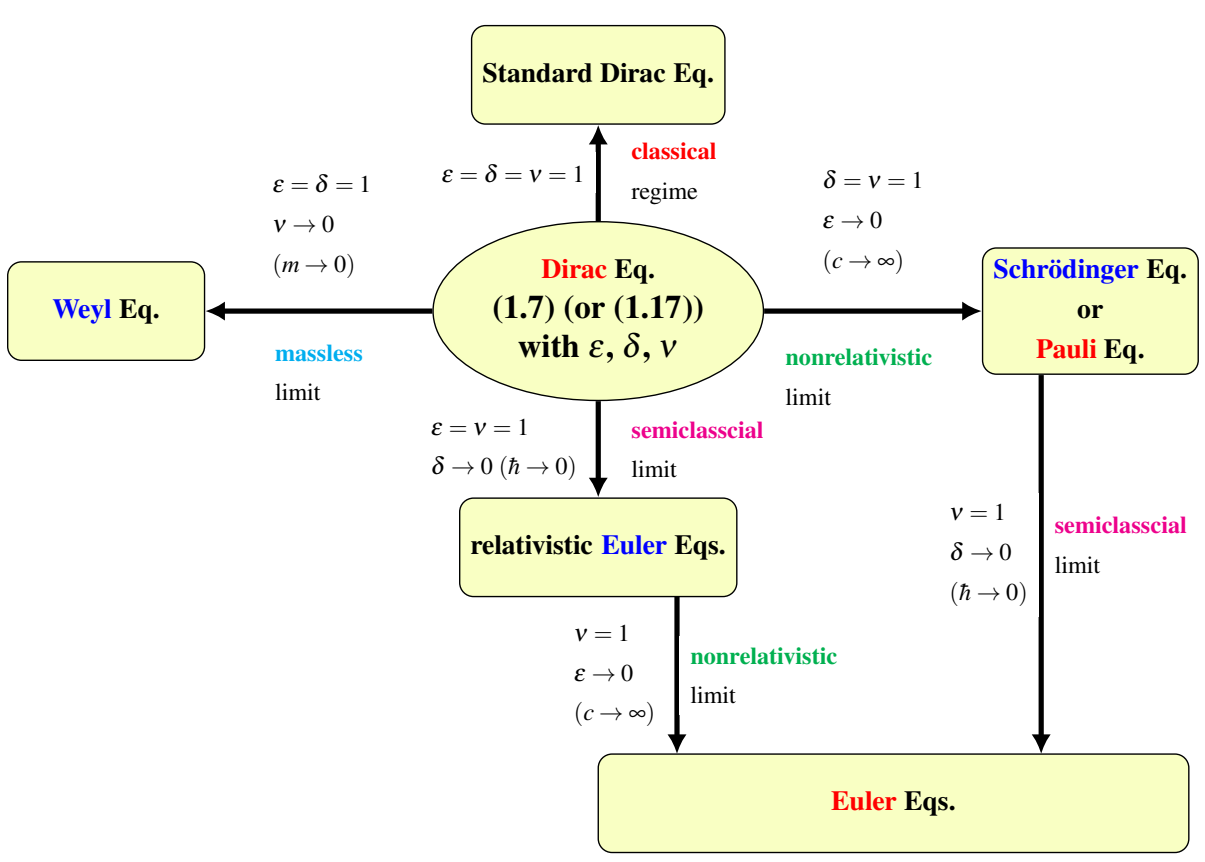

Fig. 1.1 Diagram of different parameter regimes and limits of the Dirac equation 1.7) (or 1.17).

(TSFP) method [9,42,20], exponential wave integrator Fourier pseudospectral (EWIFP) method [9], the Gaussian beam method [62], etc.

- Massless limit regime, i.e. $\varepsilon=\delta=1$ and $0<v \ll 1\left(\Longleftrightarrow x_{s}=\frac{\hbar}{m_{s} c}\right.$ and $\left.t_{s}=\frac{\hbar}{m_{s} c^{2}}\right)$, the mass of the particle is much less than the mass unit. In this parameter regime, the Dirac equation (1.7) (or (1.17) ) converges - regularly - to the Weyl equation [51,63] with linear convergence rate in terms of $v$. Any numerical methods for the Dirac equation (1.7) (or 1.17) in the standard regime can be applied in this parameter regime.

- Nonrelativistic limit regime, i.e. $\delta=v=1$ and $0<\varepsilon \ll 1\left(\Longleftrightarrow m_{s}=m\right.$ and $\left.t_{s}=\frac{m x_{s}^{2}}{\hbar}\right)$, i.e. the wave speed is much less than the speed of light. In this parameter regime, formally the dispersion relation (1.16) (or 1.25) suggests $\omega(\mathbf{k})=\varepsilon^{-2}+O(1)$ when $|\mathbf{k}|=O(1)$ and thus the solution propagates waves with wavelength at $O\left(\varepsilon^{2}\right)$ and $O(1)$ in time and space, respectively, when $0<\varepsilon \ll 1$. In addition, if the initial data $\Psi_{0}=O(1)$ in (1.8) (or $\Phi_{0}=O(1)$ in (1.18)), then the solution $\Psi=O(1)$ of 1.7) with 1.8) (or $\Phi=O(1)$ of 1.17 with $(1.18)$ ), which implies that the probability density $\rho=O(1)$ in 1.11) (or (1.21)), current density $\mathbf{J}=O\left(\varepsilon^{-1}\right)$ in 1.13) (or 1.23) and the energy $E(\Psi(t, \cdot))=O\left(\varepsilon^{-2}\right)$ in 1.10) (or $E(\Phi(t, \cdot))=O\left(\varepsilon^{-2}\right)$ in (1.20)). The highly oscillatory nature of the solution in time and the unboundedness of the energy bring significant difficulty in mathematical analysis and numerical simulation of the Dirac equation in the nonrelativistic regime, i.e. $0<\varepsilon \ll 1$. By diagonalizing the Dirac operator and using proper ansatz, one can show that the Dirac equation (1.7) (or 1.17) converges - 
singularly - to the Pauli equation [15,43] and/or the Schrödinger equation [6, 15] when $\varepsilon \rightarrow 0^{+}$. Rigorous error estimates were established for the FDTD, TSFP and EWI-FP methods in this parameter regime [9], which depend explicitly on the mesh size $h$, time step $\tau$ and the small parameter $\varepsilon$. Recently, a uniformly accurate multiscale time integrator pseudospectral method was proposed and analyzed for the Dirac equation in the nonrelativistic limit regime, which converges uniformly with respect to $\varepsilon \in(0,1]$ [8, 46].

- Semiclassical limit regime, i.e. $\varepsilon=v=1$ and $0<\delta \ll 1\left(\Longleftrightarrow m_{s}=m\right.$ and $\left.t_{s}=\frac{x_{s}}{c}\right)$, the quantum effect could be neglected. In this parameter regime, the solution propagates waves with wavelength at $O(\delta)$ in space and time [18] when $0<\delta \ll 1$. In addition, if the initial data $\Psi_{0}=O(1)$ in $(1.8)$ (or $\Phi_{0}=O(1)$ in $(1.18)$ ), then the solution $\Psi=O(1)$ of (1.7) with 1.8) (or $\Phi=O(1)$ of (1.17) with 1.18), which implies that the probability density $\rho=O(1)$ in (1.11) (or (1.21)), current density $\mathbf{J}=O(1)$ in 1.13 ) (or 1.23) and the energy $E(\Psi(t, \cdot))=O(1)$ in (1.10) (or $E(\Phi(t, \cdot))=O(1)$ in 1.20). The highly oscillatory nature of the solution in time and space brings significant difficulty in mathematical analysis and numerical simulation of the Dirac equation in the semiclassical limit regime, i.e. $0<\delta \ll 1$. By using the Wigner transformation method, one can show that the Dirac equation (1.7) (or 1.17) converges - singularly - to the relativistic Euler equations [5,39,53]. Similar to the analysis of different numerical methods for the Schrödinger equation in the semiclassical limit regime [2,7, 11, 12, 21,22, 45], it is an interesting question to establish rigorous error bounds of different numerical methods for the Dirac equation in the semiclassical limit regime such that they depend explicitly on mesh size $h$, time step $\tau$ as well as the small parameter $\delta \in(0,1]$.

- Simultaneously nonrelativistic and massless limit regimes, i.e. $\delta=1, v \sim \varepsilon$ and $0<$ $\varepsilon \ll 1$ ( $\Longleftrightarrow t_{s}=\frac{m_{s} x_{s}^{2}}{\hbar}$ ), the wave speed is much less than the speed of light and the mass of the particle is much less than the mass unit. Here we assume $v=v_{0} \varepsilon$ with $v_{0}>0$ a constant independent of $\varepsilon \in(0,1]$. In this case, the Dirac equation (1.7) can be re-written as $(d=1,2,3)$

$$
i \partial_{t} \Psi=\left(-i \frac{1}{\varepsilon} \sum_{j=1}^{d} \alpha_{j} \partial_{j}+\frac{v_{0}}{\varepsilon} \beta\right) \Psi+\left(V(\mathbf{x}) I_{4}-\sum_{j=1}^{d} A_{j}(\mathbf{x}) \alpha_{j}\right) \Psi, \quad \mathbf{x} \in \mathbb{R}^{d}
$$

and respectively, the Dirac equation (1.17) can be re-written as $(d=1,2)$

$$
i \partial_{t} \Phi=\left(-i \frac{1}{\varepsilon} \sum_{j=1}^{d} \sigma_{j} \partial_{j}+\frac{v_{0}}{\varepsilon} \sigma_{3}\right) \Phi+\left(V(\mathbf{x}) I_{2}-\sum_{j=1}^{d} A_{j}(\mathbf{x}) \sigma_{j}\right) \Phi, \quad \mathbf{x} \in \mathbb{R}^{d} .
$$

In this parameter regime, formally the dispersion relation (1.16) (or 1.25) suggests $\omega(\mathbf{k})=O\left(\varepsilon^{-1}\right)$ when $|\mathbf{k}|=O(1)$ and thus the solution propagates waves with wavelength at $O(\varepsilon)$ and $O(1)$ in time and space, respectively, when $0<\varepsilon \ll 1$. In addition, if the initial data $\Psi_{0}=O(1)$ in $(1.8)$ (or $\Phi_{0}=O(1)$ in 1.18$)$ ), then the solution $\Psi=O(1)$ of (1.26) with (1.8) (or $\Phi=O(1)$ of (1.27) with (1.18), which implies that the proba- 
bility density $\rho=O(1)$ in 1.11 (or 1.21), current density $\mathbf{J}=O\left(\varepsilon^{-1}\right)$ in 1.13) (or (1.23) and the energy $E(\Psi(t, \cdot))=O\left(\varepsilon^{-1}\right)$ in 1.10) (or $E(\Phi(t, \cdot))=O\left(\varepsilon^{-1}\right)$ in 1.20). Again, the highly oscillatory nature of the solution in time and the unboundedness of the energy bring significant difficulty in mathematical analysis and numerical simulation of the Dirac equation in this parameter regime. In fact, it is an interesting question to study the singular limit of the Dirac equation (1.26) (or (1.27) when $\varepsilon \rightarrow 0^{+}$and establish rigorous error bounds of different numerical methods for the Dirac equation in this parameter regime such that they depend explicitly on mesh size $h$, time step $\tau$ as well as the small parameter $\varepsilon \in(0,1]$.

First-order and second-order (in time) time-splitting spectral methods have been proposed and analyzed for the Dirac equation (1.7) (or (1.17) [9]. Extension to higher order, e.g. fourth-order, time-splitting spectral methods can be done straightforward by adapting the high order splitting methods [14,47,57], e.g. the standard fourth-order splitting $\left(S_{4}\right)$ [37, 55,64] or the fourth-order partitioned Runge-Kutta $\left(S_{4 \mathrm{RK}}\right)$ splitting method [16, 38]. As it was observed in the literature [47], the $S_{4}$ splitting method has to use negative time step in at least one of the sub-problems at each time interval [37, 55,64], which causes some kind of drawbacks in practical computation, and the number of sub-problems in the $S_{4 \mathrm{RK}}$ splitting method at each time interval is much bigger than that of the $S_{4}$ splitting method [16], which increases the computational cost at each time step a lot. Motivated by the fourth-order gradient symplectic integrator for the Schödinger equation invented by [23,24,25], a new fourth-order compact time-splitting $\left(S_{4 \mathrm{c}}\right)$ Fourier pseudospectral method will be proposed for the Dirac equation by splitting the Dirac equation into two parts together with using the double commutator between them to integrate the Dirac equation at each time interval. The method is explicit, fourth-order in time and spectral order in space. We compare the accuracy and efficiency as well as long time behavior of the $S_{4 \mathrm{c}}$ method with many other existing time-splitting methods for the Dirac equation. Numerical results demonstrate the advantage of the proposed $S_{4 \mathrm{c}}$ in terms of efficiency and accuracy, especially in 1D and high dimensions (2D and 3D) without magnetic potential. We also report the spatial/temporal resolution of the $S_{4 \mathrm{c}}$ method for the Dirac equation in different parameter regimes.

The rest of the paper is organized as follows. In section 2, we review different timesplitting schemes for differential equations. In section 3, we calculate the double commutator between the two parts decoupled from the Dirac equation. A fourth-order compact time-splitting Fourier pseudospectral method is proposed for the Dirac equation in section 4. In section 5, we compare accuracy and efficiency as well as long time behavior of different time-splitting methods for the Dirac equation. In section 6, we report spatial/temporal resolution of the fourth-order compact time-splitting Fourier pseudospectral method for the Dirac equation in different parameter regimes. Finally, some concluding remarks are drawn in section 7. Throughout the paper, we adopt the standard Sobolev spaces and the corre- 
sponding norms and adopt $A \lesssim B$ to mean that there exists a generic constant $C>0$ independent of $\varepsilon, \tau, h, \delta$ and $v$ such that $|A| \leq C B$.

\section{Review of different time-splitting schemes}

Splitting (or split-step or time-splitting) methods have been widely used in numerically integrating differential equations [47]. Combined with different spatial discretization schemes, they have also been applied in solving partial differential equations [47]. For details, we refer to [56,57,58] and references therein.

For simplicity of notations and the convenience of readers, here we review several timesplitting schemes for integrating a differential equation in the form

$$
\partial_{t} u(t, \mathbf{x})=(T+W) u(t, \mathbf{x})
$$

with the initial data

$$
u(0, \mathbf{x})=u_{0}(\mathbf{x})
$$

where $T$ and $W$ are two time-independent operators. For any time step $\tau>0$, formally the solution of 2.1 with 2.2 can be represented as

$$
u(\tau, \mathbf{x})=e^{\tau(T+W)} u_{0}(\mathbf{x}) .
$$

A splitting (or split-step or time-splitting) scheme can be designed by approximating the operator $e^{\tau(T+W)}$ by a product of a sequence of $e^{\tau T}$ and $e^{\tau W}$ [55,64], i.e.

$$
e^{\tau(T+W)} \approx \Pi_{j=1}^{n} e^{a_{j} \tau T} e^{b_{j} \tau W},
$$

where $n \geq 1, a_{j} \in \mathbb{R}$ and $b_{j} \in \mathbb{R}(j=1, \ldots, n)$ are to be determined such that the approximation has certain order of accuracy in terms of $\tau$ [55,64]. Without loss of generality, here we suppose that the computation for $e^{\tau W}$ is easier and/or more efficient than that for $e^{\tau T}$.

\subsection{First-order and second-order time-splitting methods}

Taking $n=1$ and $a_{1}=b_{1}=1$ in 2.4, one can obtain the first-order Lie-Trotter splitting $\left(S_{1}\right)$ method as [61]

$$
u(\tau, \mathbf{x}) \approx S_{1}(\tau) u_{0}(\mathbf{x}):=e^{\tau T} e^{\tau W} u_{0}(\mathbf{x}) .
$$

In this method, one needs to integrate the operator $T$ once and the operator $W$ once. By using Taylor expansion, one can formally show the local truncation error as [54]

$$
\left\|u(\tau, \mathbf{x})-S_{1}(\tau) u_{0}(\mathbf{x})\right\| \leq C_{1} \tau^{2}
$$


where $C_{1}>0$ is a constant independent of $\tau$ and $\|\cdot\|$ is a norm depending on the problem. Thus the method is formally a first-order integrator [47].

Similarly, taking $n=2, a_{1}=0, b_{1}=\frac{1}{2}, a_{2}=1$ and $b_{2}=\frac{1}{2}$, one can obtain the secondorder Strang splitting $\left(S_{2}\right)$ method as [54]

$$
u(\tau, \mathbf{x}) \approx S_{2}(\tau) u_{0}(\mathbf{x}):=e^{\frac{\tau}{2} W} e^{\tau T} e^{\frac{\tau}{2} W} u_{0}(\mathbf{x})
$$

In this method, one needs to integrate the operator $T$ once and the operator $W$ twice. Again, by using Taylor expansion, one can formally show the local truncation error as [54]

$$
\left\|u(\tau, \mathbf{x})-S_{2}(\tau) u_{0}(\mathbf{x})\right\| \leq C_{2} \tau^{3}
$$

where $C_{2}>0$ is a constant independent of $\tau$. Thus it is formally a second-order integrator [47.

\subsection{Fourth-order time-splitting methods}

High order, especially fourth-order, splitting methods for 2.1) with 2.2 via the construction (2.4) had been extensively studied in the literature [23, 24].

For simplicity, here we only mention a popular fourth-order Forest-Ruth (or Yoshida) splitting $\left(S_{4}\right)$ method [37, 55, 64] as

$$
u(\tau, \mathbf{x}) \approx S_{4}(\tau) u_{0}(\mathbf{x}):=S_{2}\left(w_{1} \tau\right) S_{2}\left(w_{2} \tau\right) S_{2}\left(w_{1} \tau\right) u_{0}(\mathbf{x})
$$

where

$$
w_{1}=\frac{1}{2-2^{1 / 3}}, \quad w_{2}=-\frac{2^{1 / 3}}{2-2^{1 / 3}} .
$$

In this method, one needs to integrate the operator $T$ three times and the operator $W$ four times. Again, by using Taylor expansion, one can formally show the local truncation error as [37]

$$
\left\|u(\tau, \mathbf{x})-S_{4}(\tau) u_{0}(\mathbf{x})\right\| \leq C_{4} \tau^{5},
$$

where $C_{4}>0$ is a constant independent of $\tau$. Thus it is formally a fourth-order integrator [47]. Due to that negative time steps, e.g. $w_{2}<0$, are used in the method, in general, it cannot be applied to solve dissipative differential equations. In addition, as it was noticed in the literature [47], some drawbacks of the $S_{4}$ method were reported, such as the constant $C_{4}$ is usually much larger than $C_{1}$ and $C_{2}$, and the fourth-order accuracy could be observed only when $\tau$ is very small [47,58]. 
To overcome the drawbacks of the $S_{4}$ method, the fourth-order partitioned RungeKutta splitting $\left(S_{4 \mathrm{RK}}\right)$ method was proposed [16,38] as

$$
\begin{aligned}
u(\tau, \mathbf{x}) & \approx S_{4 \mathrm{RK}}(\tau) u_{0}(\mathbf{x}) \\
& :=e^{a_{1} \tau W} e^{b_{1} \tau T} e^{a_{2} \tau W} e^{b_{2} \tau T} e^{a_{3} \tau W} e^{b_{3} \tau T} e^{a_{4} \tau W} e^{b_{3} \tau T} e^{a_{3} \tau W} e^{b_{2} \tau T} e^{a_{2} \tau W} e^{b_{1} \tau T} e^{a_{1} \tau W} u_{0}(\mathbf{x})
\end{aligned}
$$

where

$$
\begin{aligned}
& a_{1}=0.0792036964311957, \quad a_{2}=0.353172906049774, \\
& a_{3}=-0.0420650803577195, \quad a_{4}=1-2\left(a_{1}+a_{2}+a_{3}\right), \\
& b_{1}=0.209515106613362, \quad b_{2}=-0.143851773179818, \quad b_{3}=\frac{1}{2}-\left(b_{1}+b_{2}\right) .
\end{aligned}
$$

In this method, one needs to integrate the operator $T$ six times and the operator $W$ seven times. Again, by using Taylor expansion, one can formally show the local truncation error as [16]

$$
\left\|u(\tau, \mathbf{x})-S_{4 \mathrm{RK}}(\tau) u_{0}(\mathbf{x})\right\| \leq \widetilde{C}_{4} \tau^{5}
$$

where $\widetilde{C}_{4}>0$ is a constant independent of $\tau$. Thus it is formally a fourth-order integrator [47]. It is easy to see that the computational cost of the $S_{4 \mathrm{RK}}$ method is about two times that of the $S_{4}$ method. In this method, negative time steps, e.g. $a_{3}<0$, have also been used.

\subsection{Fourth-order compact time-splitting methods}

To avoid the negative time steps and motivated by the numerical integration of the Schrödinger equation [23,24,25], a fourth-order gradient symplectic integrator was proposed by S.

A. Chin [23] as

$$
u(\tau, \mathbf{x}) \approx S_{4 \mathrm{c}}(\tau) u_{0}(\mathbf{x}):=e^{\frac{1}{6} \tau W} e^{\frac{1}{2} \tau T} e^{\frac{2}{3} \tau \widehat{W}} e^{\frac{1}{2} \tau T} e^{\frac{1}{6} \tau W} u_{0}(\mathbf{x})
$$

where

$$
\widehat{W}:=W+\frac{1}{48} \tau^{2}[W,[T, W]],
$$

with $[T, W]:=T W-W T$ the commutator of the two operators $T$ and $W$ and $[W,[T, W]]$ a double commutator. Again, by using Taylor expansion, one can formally show the local truncation error as [23,24]

$$
\left\|u(\tau, \mathbf{x})-S_{4 \mathrm{c}}(\tau) u_{0}(\mathbf{x})\right\| \leq \widehat{C}_{4} \tau^{5}
$$

where $\widehat{C}_{4}>0$ is a constant independent of $\tau$. Thus it is formally a fourth-order integrator [47]. In this method, in general, one needs to integrate the operator $T$ twice and the operator $W$ three times under the assumption that the computation of $\widehat{W}$ is equivalent to that of $W$. 


\begin{tabular}{|c|ccccc|}
\hline & $S_{1}$ & $S_{2}$ & $S_{4}$ & $S_{4 \mathrm{RK}}$ & $S_{4 \mathrm{c}}$ \\
\hline $\mathrm{T}$ & 1 & 1 & 3 & 6 & 2 \\
\hline $\mathrm{W}$ & 1 & 2 & 4 & 7 & 3 \\
\hline
\end{tabular}

Table 2.1 The numbers of operators $T$ and $W$ to be implemented in different time-splitting methods.

Thus it is more efficient than the $S_{4}$ and $S_{4 \mathrm{RK}}$ methods. In this sense, it is more appropriate to name it as a fourth-order compact splitting $\left(S_{4 \mathrm{c}}\right)$ method since, at each time step, the number of sub-steps in it is much less than those in the $S_{4}$ and $S_{4 \mathrm{RK}}$ methods. Another advantage of the $S_{4 \mathrm{c}}$ method is that there is no negative time step in it.

For comparison, Table 2.1 lists the numbers of $T$ and $W$ to be integrated by different splitting methods. From it, under the assumption that the computation for $e^{\tau W}$ is easier and/or more efficient than that for $e^{\tau T}$ and the computation of $e^{\tau W}$ is similar to that for $e^{\tau W}$, we could draw the following conclusions: (i) the computational time of $S_{2}$ is almost the same as that of $S_{1}$; (ii) the computational time of $S_{4 \mathrm{c}}$ is about two times of that of $S_{2}$ (or $S_{1}$ ); (iii) among the three fourth-order splitting methods, $S_{4 \mathrm{c}}$ is the most efficient and $S_{4 \mathrm{RK}}$ is the most expensive.

\section{Computation for the double commutator $[W,[T, W]]$}

In this section, we first show that the double commutator $[W,[T, W]]$ is linear in $T$ and then compute it for the Dirac equations (1.17) for $d=1,2$ and (1.7) for $d=1,2,3$.

Lemma 1 Let $T$ and $W$ be two operators, then we have

$$
[W,[T, W]]=2 W T W-W W T-T W W .
$$

Thus the double commutator $[W,[T, W]]$ is linear in $T$, i.e. for any two operators $T_{1}$ and $T_{2}$, we have

$$
\left[W,\left[a_{1} T_{1}+a_{2} T_{2}, W\right]\right]=a_{1}\left[W,\left[T_{1}, W\right]\right]+a_{2}\left[W,\left[T_{2}, W\right]\right], \quad a_{1}, a_{2} \in \mathbb{R}
$$

Proof Noticing $[T, W]:=T W-W T$, we have

$$
\begin{aligned}
{[W,[T, W]] } & =[W,(T W-W T)]=W(T W-W T)-(T W-W T) W \\
& =W T W-W W T-T W W+W T W \\
& =2 W T W-W W T-T W W .
\end{aligned}
$$

From [3.3), it is easy to see that the double commutator $[W,[T, W]]$ is linear in $T$, i.e. (3.2) is valid. 
3.1 Double commutators of the Dirac equation in 1D

Lemma 2 For the Dirac equation (1.17) in $1 D$, i.e. $d=1$, define

$$
T=-\frac{1}{\varepsilon} \sigma_{1} \partial_{1}-\frac{i v}{\delta \varepsilon^{2}} \sigma_{3}, \quad W=-\frac{i}{\delta}\left(V(x) I_{2}-A_{1}(x) \sigma_{1}\right),
$$

we have

$$
[W,[T, W]]=-\frac{4 i v}{\delta^{3} \varepsilon^{2}} A_{1}^{2}(x) \sigma_{3} .
$$

Proof Combining (3.4) and 3.2), we obtain

$$
[W,[T, W]]=-\frac{1}{\varepsilon}\left[W,\left[\sigma_{1} \partial_{1}, W\right]\right]-\frac{i v}{\delta \varepsilon^{2}}\left[W,\left[\sigma_{3}, W\right]\right] .
$$

Noticing (3.1) and (3.4), we have

$$
\begin{aligned}
{\left[W,\left[\sigma_{1} \partial_{1}, W\right]\right]=} & 2\left(-\frac{i}{\delta}\left(V(x) I_{2}-A_{1}(x) \sigma_{1}\right)\right)\left(\sigma_{1} \partial_{1}\right)\left(-\frac{i}{\delta}\left(V(x) I_{2}-A_{1}(x) \sigma_{1}\right)\right) \\
& -\left(-\frac{i}{\delta}\left(V(x) I_{2}-A_{1}(x) \sigma_{1}\right)\right)^{2}\left(\sigma_{1} \partial_{1}\right)-\left(\sigma_{1} \partial_{1}\right)\left(-\frac{i}{\delta}\left(V(x) I_{2}-A_{1}(x) \sigma_{1}\right)\right)^{2} \\
= & -\frac{2}{\delta^{2}}\left(V(x) I_{2}-A_{1}(x) \sigma_{1}\right) \sigma_{1} \partial_{1}\left(V(x) I_{2}-A_{1}(x) \sigma_{1}\right) \\
& +\frac{1}{\delta^{2}}\left(V(x) I_{2}-A_{1}(x) \sigma_{1}\right)^{2} \sigma_{1} \partial_{1}+\frac{1}{\delta^{2}} \sigma_{1} \partial_{1}\left(V(x) I_{2}-A_{1}(x) \sigma_{1}\right)^{2} \\
= & -\frac{2}{\delta^{2}} \sigma_{1}\left(V(x) I_{2}-A_{1}(x) \sigma_{1}\right) \partial_{1}\left(V(x) I_{2}-A_{1}(x) \sigma_{1}\right) \\
& -\frac{2}{\delta^{2}} \sigma_{1}\left(V(x) I_{2}-A_{1}(x) \sigma_{1}\right)^{2} \partial_{1}+\frac{2}{\delta^{2}} \sigma_{1}\left(V(x) I_{2}-A_{1}(x) \sigma_{1}\right)^{2} \partial_{1} \\
& +\frac{2}{\delta^{2}} \sigma_{1}\left(V(x) I_{2}-A_{1}(x) \sigma_{1}\right) \partial_{1}\left(V(x) I_{2}-A_{1}(x) \sigma_{1}\right) \\
= & 0 . \\
{\left[W,\left[\sigma_{3}, W\right]\right]=2 } & \left(-\frac{i}{\delta}\left(V(x) I_{2}-A_{1}(x) \sigma_{1}\right)\right) \sigma_{3}\left(-\frac{i}{\delta}\left(V(x) I_{2}-A_{1}(x) \sigma_{1}\right)\right) \\
- & \left(-\frac{i}{\delta}\left(V(x) I_{2}-A_{1}(x) \sigma_{1}\right)\right)^{2} \sigma_{3}-\sigma_{3}\left(-\frac{i}{\delta}\left(V(x) I_{2}-A_{1}(x) \sigma_{1}\right)\right)^{2} \\
=- & \frac{2}{\delta^{2}}\left(V(x) I_{2}-A_{1}(x) \sigma_{1}\right)\left(V(x) I_{2}+A_{1}(x) \sigma_{1}\right) \sigma_{3}+\frac{1}{\delta^{2}}\left(V(x) I_{2}-A_{1}(x) \sigma_{1}\right)^{2} \sigma_{3} \\
+ & \frac{1}{\delta^{2}}\left(V(x) I_{2}+A_{1}(x) \sigma_{1}\right)^{2} \sigma_{3} \\
= & \frac{1}{\delta^{2}}\left(2 V^{2}(x) I_{2}-2 A_{1}^{2}(x) I_{2}-\left(V^{2}(x) I_{2}+A_{1}^{2}(x) I_{2}-2 A_{1}(x) V(x) \sigma_{1}\right)\right. \\
& \frac{1}{\delta^{2}}\left(-4 A_{1}^{2}(x) I_{2}\right) \sigma_{3}=\frac{4}{\delta^{2}} A_{1}^{2}(x) \sigma_{3} . \\
& \left.\left.(3.8) V(x) \sigma_{1}\right)\right) \sigma_{3} \\
& \\
& \\
&
\end{aligned}
$$


Plugging (3.7) and (3.8) into (3.6), we can obtain (3.5) immediately.

Combining (3.5), 3.4) and 2.15), we have

$$
\widehat{W}=W+\frac{1}{48} \tau^{2}[W,[T, W]]=-\frac{i}{\delta}\left(V(x) I_{2}-A_{1}(x) \sigma_{1}\right)-\frac{i \nu \tau^{2}}{12 \delta^{3} \varepsilon^{2}} A_{1}^{2}(x) \sigma_{3},
$$

which immediately implies that the computation of $e^{\tau \widehat{W}}$ is similar (or at almost the same computational cost) to that for $e^{\tau W}$ in this case.

Corollary 1 For the Dirac equation (1.7) in $1 D$, i.e. $d=1$, define

$$
T=-\frac{1}{\varepsilon} \alpha_{1} \partial_{1}-\frac{i \nu}{\delta \varepsilon^{2}} \beta, \quad W=-\frac{i}{\delta}\left(V(x) I_{4}-A_{1}(x) \alpha_{1}\right),
$$

we have

$$
[W,[T, W]]=-\frac{4 i v}{\delta^{3} \varepsilon^{2}} A_{1}^{2}(x) \beta
$$

3.2 Double commutators of the Dirac equation in 2D and 3D

Similar to the 1D case, we have (see detailed computation in Appendix A)

Lemma 3 For the Dirac equation (1.17) in 2D, i.e. $d=2$, define

$$
T=-\frac{1}{\varepsilon} \sigma_{1} \partial_{1}-\frac{1}{\varepsilon} \sigma_{2} \partial_{2}-\frac{i \nu}{\delta \varepsilon^{2}} \sigma_{3}, \quad W=-\frac{i}{\delta}\left(V(\mathbf{x}) I_{2}-A_{1}(\mathbf{x}) \sigma_{1}-A_{2}(\mathbf{x}) \sigma_{2}\right)
$$

we have

$$
[W,[T, W]]=F_{3}(\mathbf{x})+F_{1}(\mathbf{x}) \partial_{1}+F_{2}(\mathbf{x}) \partial_{2},
$$

where

$$
\begin{aligned}
F_{1}(\mathbf{x})= & \frac{4}{\delta^{2} \varepsilon}\left(-A_{2}^{2}(\mathbf{x}) \sigma_{1}+A_{1}(\mathbf{x}) A_{2}(\mathbf{x}) \sigma_{2}\right), \quad F_{2}(\mathbf{x})=\frac{4}{\delta^{2} \varepsilon}\left(A_{1}(\mathbf{x}) A_{2}(\mathbf{x}) \sigma_{1}-A_{1}^{2}(\mathbf{x}) \sigma_{2}\right), \\
F_{3}(\mathbf{x})= & \frac{4}{\delta^{2} \varepsilon}\left(A_{1}(\mathbf{x}) \partial_{2} A_{2}(\mathbf{x})-A_{2}(\mathbf{x}) \partial_{1} A_{2}(\mathbf{x})\right) \sigma_{1}+\frac{4}{\delta^{2} \varepsilon}\left(A_{2}(\mathbf{x}) \partial_{1} A_{1}(\mathbf{x})-A_{1}(\mathbf{x}) \partial_{2} A_{1}(\mathbf{x})\right) \sigma_{2} \\
& +\frac{4 i}{\delta^{2} \varepsilon}\left(A_{2}(\mathbf{x}) \partial_{1} V(\mathbf{x})-A_{1}(\mathbf{x}) \partial_{2} V(\mathbf{x})-\frac{v}{\delta \varepsilon}\left(A_{1}^{2}(\mathbf{x})+A_{2}^{2}(\mathbf{x})\right)\right) \sigma_{3} .
\end{aligned}
$$

Corollary 2 For the Dirac equation (1.7) in 2D, i.e. $d=2$, define

$$
T=-\frac{1}{\varepsilon} \alpha_{1} \partial_{1}-\frac{1}{\varepsilon} \alpha_{2} \partial_{2}-\frac{i \nu}{\delta \varepsilon^{2}} \beta, \quad W=-\frac{i}{\delta}\left(V(\mathbf{x}) I_{2}-A_{1}(\mathbf{x}) \alpha_{1}-A_{2}(\mathbf{x}) \alpha_{2}\right),
$$

we have

$$
[W,[T, W]]=F_{3}(\mathbf{x})+F_{1}(\mathbf{x}) \partial_{1}+F_{2}(\mathbf{x}) \partial_{2},
$$


where

$$
\begin{aligned}
F_{1}(\mathbf{x})= & \frac{4}{\delta^{2} \varepsilon}\left(-A_{2}^{2}(\mathbf{x}) \alpha_{1}+A_{1}(\mathbf{x}) A_{2}(\mathbf{x}) \alpha_{2}\right), \quad F_{2}(\mathbf{x})=\frac{4}{\delta^{2} \varepsilon}\left(A_{1}(\mathbf{x}) A_{2}(\mathbf{x}) \alpha_{1}-A_{1}^{2}(\mathbf{x}) \alpha_{2}\right), \\
F_{3}(\mathbf{x})= & \frac{4}{\delta^{2} \varepsilon}\left(A_{1}(\mathbf{x}) \partial_{2} A_{2}(\mathbf{x})-A_{2}(\mathbf{x}) \partial_{1} A_{2}(\mathbf{x})\right) \alpha_{1}+\frac{4}{\delta^{2} \varepsilon}\left(A_{2}(\mathbf{x}) \partial_{1} A_{1}(\mathbf{x})-A_{1}(\mathbf{x}) \partial_{2} A_{1}(\mathbf{x})\right) \alpha_{2} \\
& +\frac{4 i}{\delta^{2} \varepsilon}\left(A_{2}(\mathbf{x}) \partial_{1} V(\mathbf{x})-A_{1}(\mathbf{x}) \partial_{2} V(\mathbf{x})\right) \gamma \alpha_{3}-\frac{4 i \nu}{\delta^{3} \varepsilon^{2}}\left(A_{1}^{2}(\mathbf{x})+A_{2}^{2}(\mathbf{x})\right) \beta,
\end{aligned}
$$

where

$$
\gamma=\left(\begin{array}{cc}
\mathbf{0} & I_{2} \\
I_{2} & \mathbf{0}
\end{array}\right)
$$

For the Dirac equation (1.7) in 3D, i.e. $d=3$, we have (see detailed computation in Appendix B)

Lemma 4 For the Dirac equation (1.7) in 3D, i.e. $d=3$, define

$$
T=-\frac{1}{\varepsilon} \sum_{j=1}^{3} \alpha_{j} \partial_{j}-\frac{i \nu}{\delta \varepsilon^{2}} \beta, \quad W=-\frac{i}{\delta}\left(V(\mathbf{x}) I_{4}-\sum_{j=1}^{3} A_{j}(\mathbf{x}) \alpha_{j}\right),
$$

we have

$$
[W,[T, W]]=F_{4}(\mathbf{x})+F_{1}(\mathbf{x}) \partial_{1}+F_{2}(\mathbf{x}) \partial_{2}+F_{3}(\mathbf{x}) \partial_{3},
$$

where

$$
\begin{aligned}
F_{1}(\mathbf{x})= & \frac{4}{\delta^{2} \varepsilon}\left(-\left(A_{2}^{2}(\mathbf{x})+A_{3}^{2}(\mathbf{x})\right) \alpha_{1}+A_{1}(\mathbf{x}) A_{2}(\mathbf{x}) \alpha_{2}+A_{1}(\mathbf{x}) A_{3}(\mathbf{x}) \alpha_{3}\right) \\
F_{2}(\mathbf{x})= & \frac{4}{\delta^{2} \varepsilon}\left(A_{2}(\mathbf{x}) A_{1}(\mathbf{x}) \alpha_{1}-\left(A_{1}^{2}(\mathbf{x})+A_{3}^{2}(\mathbf{x})\right) \alpha_{2}+A_{2}(\mathbf{x}) A_{3}(\mathbf{x}) \alpha_{3}\right) \\
F_{3}(\mathbf{x})= & \frac{4}{\delta^{2} \varepsilon}\left(A_{3}(\mathbf{x}) A_{1}(\mathbf{x}) \alpha_{1}+A_{3}(\mathbf{x}) A_{2}(\mathbf{x}) \alpha_{2}-\left(A_{1}^{2}(\mathbf{x})+A_{2}^{2}(\mathbf{x})\right) \alpha_{3}\right) \\
F_{4}(\mathbf{x})= & \frac{4}{\delta^{2} \varepsilon}\left(A_{1}(\mathbf{x})\left(\partial_{2} A_{2}(\mathbf{x})+\partial_{3} A_{3}(\mathbf{x})\right)-A_{2}(\mathbf{x}) \partial_{1} A_{2}(\mathbf{x})-A_{3}(\mathbf{x}) \partial_{1} A_{3}(\mathbf{x})\right) \alpha_{1} \\
& +\frac{4}{\delta^{2} \varepsilon}\left(A_{2}(\mathbf{x})\left(\partial_{1} A_{1}(\mathbf{x})+\partial_{3} A_{3}(\mathbf{x})\right)-A_{1}(\mathbf{x}) \partial_{2} A_{1}(\mathbf{x})-A_{3}(\mathbf{x}) \partial_{2} A_{3}(\mathbf{x})\right) \alpha_{2} \\
& +\frac{4}{\delta^{2} \varepsilon}\left(A_{3}(\mathbf{x})\left(\partial_{1} A_{1}(\mathbf{x})+\partial_{2} A_{2}(\mathbf{x})\right)-A_{1}(\mathbf{x}) \partial_{3} A_{1}(\mathbf{x})-A_{2}(\mathbf{x}) \partial_{3} A_{2}(\mathbf{x})\right) \alpha_{3} \\
& +\frac{4 i}{\delta^{2} \varepsilon}\left(A_{1}(\mathbf{x})\left(\partial_{2} A_{3}(\mathbf{x})-\partial_{3} A_{2}(\mathbf{x})\right)+A_{2}(\mathbf{x})\left(\partial_{3} A_{1}(\mathbf{x})-\partial_{1} A_{3}(\mathbf{x})\right)\right. \\
& \left.+A_{3}(\mathbf{x})\left(\partial_{1} A_{2}(\mathbf{x})-\partial_{2} A_{1}(\mathbf{x})\right)\right) \gamma+\frac{4 i}{\delta^{2} \varepsilon}\left(A_{3}(\mathbf{x}) \partial_{2} V(\mathbf{x})-A_{2}(\mathbf{x}) \partial_{3} V(\mathbf{x})\right) \gamma \alpha_{1} \\
& +\frac{4 i}{\delta^{2} \varepsilon}\left(A_{1}(\mathbf{x}) \partial_{3} V(\mathbf{x})-A_{3}(\mathbf{x}) \partial_{1} V(\mathbf{x})\right) \gamma \alpha_{2} \\
& +\frac{4 i}{\delta^{2} \varepsilon}\left(A_{2}(\mathbf{x}) \partial_{1} V(\mathbf{x})-A_{1}(\mathbf{x}) \partial_{2} V(\mathbf{x})\right) \gamma \alpha_{3}-\frac{4 i v}{\delta^{3} \varepsilon^{2}}\left(A_{1}^{2}(\mathbf{x})+A_{2}^{2}(\mathbf{x})+A_{3}^{2}(\mathbf{x})\right) \beta .
\end{aligned}
$$

From Lemmas 2, 3 and 4 and Corollaries 1 and 2, it is easy to observe that the double commutator will vanish when the Dirac equation 1.17) (or 1.7) has no magnetic potentials. 
Lemma 5 For the Dirac equation (1.17) in $1 D$ and $2 D$, and for the Dirac equation (1.7) in $1 D, 2 D$ and $3 D$, when there is no magnetic potential, i.e., when $A_{1}(\mathbf{x})=A_{2}(\mathbf{x})=A_{3}(\mathbf{x}) \equiv 0$, we have

$$
[W,[T, W]]=0
$$

\section{A fourth-order compact time-splitting Fourier pseudospectral method}

In this section, we present a fourth-order compact time-splitting Fourier pseudospectral method for the Dirac equation (1.7) (or 1.17) by using the $S_{4 \mathrm{c}}$ method 2.14 for time integration followed by the Fourier pseudospectral method for spatial discretization.

\subsection{Time integration by the $S_{4 \mathrm{c}}$ method in 1D}

For simplicity of notations, we present the numerical method for 1.17) in 1D first. Similar to most works in the literatures for the analysis and computation of the Dirac equation (cf. [8, 9, 10, 13] and references therein), in practical computation, we truncate the whole space problem onto an interval $\Omega=(a, b)$ with periodic boundary conditions. The truncated interval is large enough such that the truncation error is negligible. In $1 \mathrm{D}$, the Dirac equation 1.17 with periodic boundary conditions collapses to

$$
\begin{aligned}
& i \delta \partial_{t} \Phi=\left(-i \frac{\delta}{\varepsilon} \sigma_{1} \partial_{x}+\frac{v}{\varepsilon^{2}} \sigma_{3}\right) \Phi+\left(V(x) I_{2}-A_{1}(x) \sigma_{1}\right) \Phi, \quad x \in \Omega, \quad t>0 \\
& \Phi(t, a)=\Phi(t, b), \quad \partial_{x} \Phi(t, a)=\partial_{x} \Phi(t, b), \quad t \geq 0 \\
& \Phi(0, x)=\Phi_{0}(x), \quad a \leq x \leq b
\end{aligned}
$$

where $\Phi:=\Phi(t, x), \Phi_{0}(a)=\Phi_{0}(b)$ and $\Phi_{0}^{\prime}(a)=\Phi_{0}^{\prime}(b)$.

Choose a time step $\tau>0$, denote $t_{n}=n \tau$ for $n \geq 0$ and let $\Phi^{n}(x)$ be an approximation of $\Phi\left(t_{n}, x\right)$. Re-writing the Dirac equation (4.1) as

$$
\partial_{t} \Phi=\left(-\frac{1}{\varepsilon} \sigma_{1} \partial_{x}-\frac{i v}{\delta \varepsilon^{2}} \sigma_{3}\right) \Phi-\frac{i}{\delta}\left(V(x) I_{2}-A_{1}(x) \sigma_{1}\right) \Phi:=(T+W) \Phi,
$$

then we can apply the $S_{4 \mathrm{c}}$ method (2.14) for time integration over the time interval $\left[t_{n}, t_{n+1}\right]$ as

$$
\Phi^{n+1}(x)=S_{4 \mathrm{c}}(\tau) \Phi^{n}(x):=e^{\frac{1}{6} \tau W} e^{\frac{1}{2} \tau T} e^{\frac{2}{3} \tau \widehat{W}} e^{\frac{1}{2} \tau T} e^{\frac{1}{6} \tau W} \Phi^{n}(x), \quad a \leq x \leq b, \quad n \geq 0,
$$

where the two operators $T$ and $W$ are given in (3.4) and the operator $\widehat{W}$ is given in (3.9). In order to calculate $e^{\frac{1}{2} \tau T}$, we can discretize it in space via Fourier spectral method and then integrate (in phase space or Fourier space) in time exactly [9]. Since $W$ is diagonalizable 
[9], $e^{\frac{1}{6} \tau W}$ can be evaluated very efficiently [9]. For $e^{\frac{2}{3} \tau \widehat{W}}$, plugging (1.3) into $(3.9$ ), we can diagonalize it as

$$
\widehat{W}=-\frac{i}{\delta}\left(V(x) I_{2}-A_{1}(x) \sigma_{1}\right)-\frac{i v \tau^{2}}{12 \delta^{3} \varepsilon^{2}} A_{1}^{2}(x) \sigma_{3}=-i P_{2}(x) \Lambda_{2}(x) P_{2}(x)^{*}:=\widehat{W}(x),
$$

where $\Lambda_{2}(x)=\operatorname{diag}\left(\lambda_{+}^{(2)}(x), \lambda_{-}^{(2)}(x)\right)$ with $\lambda_{ \pm}^{(2)}(x)=\frac{V(x)}{\delta} \pm \frac{A_{1}(x)}{12 \delta^{3} \varepsilon^{2}} \sqrt{144 \delta^{4} \varepsilon^{4}+v^{2} \tau^{4} A_{1}^{2}(x)}$ and

$$
P_{2}(x)=\frac{1}{\sqrt{2 \beta_{1}(x)}}\left(\begin{array}{ll}
\sqrt{\beta_{1}(x)+\beta_{2}(x)} & \sqrt{\beta_{1}(x)-\beta_{2}(x)} \\
-\sqrt{\beta_{1}(x)-\beta_{2}(x)} & \sqrt{\beta_{1}(x)+\beta_{2}(x)}
\end{array}\right), \quad a \leq x \leq b,
$$

with

$$
\beta_{1}(x)=\sqrt{144 \delta^{4} \varepsilon^{4}+v^{2} \tau^{4} A_{1}^{2}(x)}, \quad \beta_{2}(x)=v \tau^{2} A_{1}(x), \quad a \leq x \leq b .
$$

Thus we have

$$
e^{\frac{2}{3} \tau \widehat{W}}=e^{-\frac{2 i}{3} \tau P_{2}(x) \Lambda_{2}(x) P_{2}(x)^{*}}=P_{2}(x) e^{-\frac{2 i}{3} \tau \Lambda_{2}(x)} P_{2}(x)^{*}, \quad a \leq x \leq b .
$$

\subsection{Full discretization in 1D}

Choose a mesh size $h:=\Delta x=\frac{b-a}{M}$ with $M$ being an even positive integer and denote the grid points as $x_{j}:=a+j h$, for $j=0,1, \ldots, M$. Denote $X_{M}=\left\{U=\left(U_{0}, U_{1}, \ldots, U_{M}\right)^{T} \mid U_{j} \in\right.$ $\left.\mathbb{C}^{2}, j=0,1, \ldots, M, U_{0}=U_{M}\right\}$. For any $U \in X_{M}$, we denote its Fourier representation as

$$
U_{j}=\sum_{l=-M / 2}^{M / 2-1} \widetilde{U}_{l} e^{i \mu_{l}\left(x_{j}-a\right)}=\sum_{l=-M / 2}^{M / 2-1} \widetilde{U}_{l} e^{2 i j l \pi / M}, \quad j=0,1, \ldots, M
$$

where $\mu_{l}$ and $\widetilde{U}_{l} \in \mathbb{C}^{2}$ are defined as

$$
\mu_{l}=\frac{2 l \pi}{b-a}, \quad \widetilde{U}_{l}=\frac{1}{M} \sum_{j=0}^{M-1} U_{j} e^{-2 i j l \pi / M}, \quad l=-\frac{M}{2}, \ldots, \frac{M}{2}-1 .
$$

For $U \in X_{M}$ and $u(x) \in L^{2}(\Omega)$, their $l^{2}$-norms are defined as

$$
\|U\|_{l^{2}}^{2}:=h \sum_{j=0}^{M-1}\left|U_{j}\right|^{2}, \quad\|u\|_{l^{2}}^{2}:=h \sum_{j=0}^{M-1}\left|u\left(x_{j}\right)\right|^{2} .
$$

Let $\Phi_{j}^{n}$ be the numerical approximation of $\Phi\left(t_{n}, x_{j}\right)$ and denote $\Phi^{n}=\left(\Phi_{0}^{n}, \Phi_{1}^{n}, \ldots, \Phi_{M}^{n}\right)^{T} \in$ $X_{M}$ as the solution vector at $t=t_{n}$. Take $\Phi_{j}^{0}=\Phi_{0}\left(x_{j}\right)$ for $j=0, \ldots, M$, then a fourth-order compact time-splitting Fourier pseudospectral $\left(S_{4 \mathrm{c}}\right)$ discretization for the Dirac equation 
(4.1) is given as

$$
\begin{aligned}
\Phi_{j}^{(1)} & =e^{\frac{\tau}{6} W\left(x_{j}\right)} \Phi_{j}^{n}=P_{1} e^{-\frac{i \tau}{6} \Lambda_{1}\left(x_{j}\right)} P_{1}^{*} \Phi_{j}^{n}, \\
\Phi_{j}^{(2)} & =\sum_{l=-M / 2}^{M / 2-1} e^{\tau \Gamma_{l}}\left(\widetilde{\Phi^{(1)}}\right)_{l} e^{i \mu_{l}\left(x_{j}-a\right)}=\sum_{l=-M / 2}^{M / 2-1} Q_{l} e^{-i \tau D_{l}} Q_{l}^{*}\left(\widetilde{\Phi^{(1)}}\right)_{l} e^{2 i j l \pi / M}, \\
\Phi_{j}^{(3)} & =e^{\frac{2 \tau}{3} \widehat{W}\left(x_{j}\right)} \Phi_{j}^{(2)}=P_{2}\left(x_{j}\right) e^{-\frac{2 i \tau}{3} \Lambda_{2}\left(x_{j}\right)} P_{2}\left(x_{j}\right)^{*} \Phi_{j}^{(2)}, \quad j=0,1, \ldots, M, \\
\Phi_{j}^{(4)} & =\sum_{l=-M / 2}^{M / 2-1} e^{\tau \Gamma_{l}}\left(\widetilde{\Phi^{(3)}}\right)_{l} e^{i \mu_{l}\left(x_{j}-a\right)}=\sum_{l=-M / 2}^{M / 2-1} Q_{l} e^{-i \tau D_{l}} Q_{l}^{*}\left(\widetilde{\Phi^{(3)}}\right)_{l} e^{2 i j l \pi / M}, \\
\Phi_{j}^{n+1} & =e^{\frac{\tau}{6} W\left(x_{j}\right)} \Phi_{j}^{(4)}=P_{1} e^{-\frac{i \tau}{6} \Lambda_{1}\left(x_{j}\right)} P_{1}^{*} \Phi_{j}^{(4)},
\end{aligned}
$$

where

$$
\begin{aligned}
& W\left(x_{j}\right):=-\frac{i}{\delta}\left(V\left(x_{j}\right) I_{2}-A_{1}\left(x_{j}\right) \sigma_{1}\right)=-i P_{1} \Lambda_{1}\left(x_{j}\right) P_{1}^{*}, \quad j=0,1, \ldots, M, \\
& \Gamma_{l}=-\frac{i \mu_{l}}{\varepsilon} \sigma_{1}-\frac{i v}{\delta \varepsilon^{2}} \sigma_{3}=-i Q_{l} D_{l} Q_{l}^{*}, \quad l=-\frac{M}{2}, \ldots, \frac{M}{2}-1,
\end{aligned}
$$

with $D_{l}=\operatorname{diag}\left(\frac{1}{\delta \varepsilon^{2}} \sqrt{v^{2}+\delta^{2} \varepsilon^{2} \mu_{l}^{2}},-\frac{1}{\delta \varepsilon^{2}} \sqrt{v^{2}+\delta^{2} \varepsilon^{2} \mu_{l}^{2}}\right), \Lambda_{1}(x)=\operatorname{diag}\left(\lambda_{+}^{(1)}(x), \lambda_{-}^{(1)}(x)\right)$ with $\lambda_{ \pm}^{(1)}(x)=\frac{1}{\delta}\left(V(x) \pm A_{1}(x)\right), \eta_{l}=\sqrt{v^{2}+\delta^{2} \varepsilon^{2} \mu_{l}^{2}}$, and

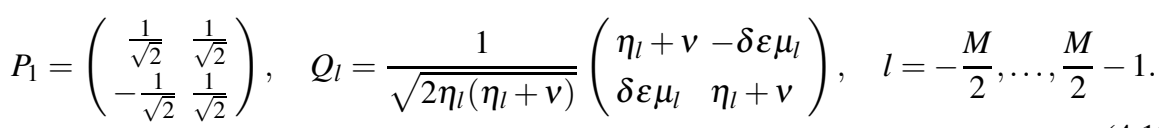

We remark here that full discretization by other time-splitting methods together with Fourier pseudospectral method for spatial discretization can be implemented similarly [9] and the details are omitted here for brevity.

\subsection{Mass conservation in 1D}

The $S_{4 \mathrm{c}}$ method (4.11) is explicit, its memory cost is $O(M)$ and its computational cost per time step is $O(M \ln M)$, it is fourth-order accurate in time and spectral accurate in space. In addition, it conserves the total probability in the discretized level, as shown in the following lemma.

Lemma 6 For any $\tau>0$, the $S_{4 c}$ method (4.11) conserves the mass in the discretized level, i.e.

$$
\left\|\Phi^{n+1}\right\|_{l^{2}}^{2}:=h \sum_{j=0}^{M-1}\left|\Phi_{j}^{n+1}\right|^{2} \equiv h \sum_{j=0}^{M-1}\left|\Phi_{j}^{0}\right|^{2}=h \sum_{j=0}^{M-1}\left|\Phi_{0}\left(x_{j}\right)\right|^{2}=\left\|\Phi_{0}\right\|_{l^{2}}^{2}, \quad n \geq 0 .
$$


Proof Noticing $W\left(x_{j}\right)^{*}=-W\left(x_{j}\right)$ and thus $\left(e^{\frac{\tau}{6} W\left(x_{j}\right)}\right)^{*} e^{\frac{\tau}{6} W\left(x_{j}\right)}=I_{2}$, from (4.11) and summing for $j=0,1, \ldots, M-1$, we get

$$
\begin{aligned}
\left\|\Phi^{n+1}\right\|_{l^{2}}^{2} & =h \sum_{j=0}^{M-1}\left|\Phi_{j}^{n+1}\right|^{2}=h \sum_{j=0}^{M-1}\left|e^{\frac{\tau}{6} W\left(x_{j}\right)} \Phi_{j}^{(4)}\right|^{2}=h \sum_{j=0}^{M-1}\left(\Phi_{j}^{(4)}\right)^{*}\left(e^{\frac{\tau}{6} W\left(x_{j}\right)}\right)^{*} e^{\frac{\tau}{6} W\left(x_{j}\right)} \Phi_{j}^{(4)} \\
& =h \sum_{j=0}^{M-1}\left(\Phi_{j}^{(4)}\right)^{*} I_{2} \Phi_{j}^{(4)}=h \sum_{j=0}^{M-1}\left|\Phi_{j}^{(4)}\right|^{2}=\left\|\Phi^{(4)}\right\|_{l^{2}}^{2}, \quad n \geq 0 .
\end{aligned}
$$

Similarly, we have

$$
\left\|\Phi^{(3)}\right\|_{l^{2}}^{2}=\left\|\Phi^{(2)}\right\|_{l^{2}}^{2}, \quad\left\|\Phi^{(1)}\right\|_{l^{2}}^{2}=\left\|\Phi^{n}\right\|_{l^{2}}^{2}, \quad n \geq 0 .
$$

Similarly, using the Parsval's identity and noticing $\Gamma_{l}^{*}=-\Gamma_{l}$ and thus $\left(e^{\tau \Gamma_{l}}\right)^{*} e^{\tau \Gamma_{l}}=I_{2}$, we get

$$
\left\|\Phi^{(4)}\right\|_{l^{2}}^{2}=\left\|\Phi^{(3)}\right\|_{l^{2}}^{2}, \quad\left\|\Phi^{(2)}\right\|_{l^{2}}^{2}=\left\|\Phi^{(1)}\right\|_{l^{2}}^{2} .
$$

Combining 4.15, 4.16) and 4.17, we obtain

$$
\left\|\Phi^{n+1}\right\|_{l^{2}}^{2}=\left\|\Phi^{(4)}\right\|_{l^{2}}^{2}=\left\|\Phi^{(3)}\right\|_{l^{2}}^{2}=\left\|\Phi^{(2)}\right\|_{l^{2}}^{2}=\left\|\Phi^{(1)}\right\|_{l^{2}}^{2}=\left\|\Phi^{n}\right\|_{l^{2}}^{2}, \quad n \geq 0 .
$$

Using the mathematical induction, we get the mass conservation (4.14).

\subsection{Discussion on extension to 2D and 3D}

When there is no magnetic potential, i.e., when $A_{1}(\mathbf{x})=A_{2}(\mathbf{x})=A_{3}(\mathbf{x}) \equiv 0$ in the Dirac equation (1.17) in $2 \mathrm{D}$ and $1.7 \mathrm{P}$ in $2 \mathrm{D}$ and $3 \mathrm{D}$, from Lemma 5 , we know that the double commutator $[W,[T, W]]=0$. In this case, noting 2.15 , we have

$$
\widehat{W}=W+\frac{1}{48} \tau^{2}[W,[T, W]]=W .
$$

Then the $S_{4 \mathrm{c}}$ method (2.14) collapses to

$$
u(\tau, \mathbf{x}) \approx S_{4 \mathrm{c}}(\tau) u_{0}(\mathbf{x}):=e^{\frac{1}{6} \tau W} e^{\frac{1}{2} \tau T} e^{\frac{2}{3} \tau W} e^{\frac{1}{2} \tau T} e^{\frac{1}{6} \tau W} u_{0}(\mathbf{x})
$$

Applying the $S_{4 \mathrm{c}}$ method 4.20 to integrate the Dirac equation 1.17) in 2D over the time interval $\left[t_{n}, t_{n+1}\right]$ with $\Phi\left(t_{n}, \mathbf{x}\right)=\Phi^{n}(\mathbf{x})$ given, we obtain

$$
\Phi^{n+1}(\mathbf{x})=S_{4 \mathrm{c}}(\tau) \Phi^{n}(\mathbf{x})=e^{\frac{1}{6} \tau W} e^{\frac{1}{2} \tau T} e^{\frac{2}{3} \tau W} e^{\frac{1}{2} \tau T} e^{\frac{1}{6} \tau W} \Phi^{n}(\mathbf{x}), \quad \mathbf{x} \in \Omega, \quad n \geq 0,
$$

where $T$ and $W$ are given in (3.12). Similarly, applying the $S_{4 \mathrm{c}}$ method (4.20) to integrate the Dirac equation (1.7) in 2D and 3D over the time interval $\left[t_{n}, t_{n+1}\right]$ with $\Psi\left(t_{n}, \mathbf{x}\right)=\Psi^{n}(\mathbf{x})$ 
given, we obtain

$$
\Psi^{n+1}(\mathbf{x})=S_{4 \mathrm{c}}(\tau) \Psi^{n}(\mathbf{x})=e^{\frac{1}{6} \tau W} e^{\frac{1}{2} \tau T} e^{\frac{2}{3} \tau W} e^{\frac{1}{2} \tau T} e^{\frac{1}{6} \tau W} \Psi^{n}(\mathbf{x}), \quad \mathbf{x} \in \Omega, \quad n \geq 0,
$$

where $T$ and $W$ are given in (3.14) and 3.17) for 2D and 3D, respectively. In practical computation, the operators $e^{\frac{1}{6} \tau W}$ and $e^{\frac{2}{3} \tau W}$ in 4.21) and 4.22) can be evaluated in physical space directly and easily [9]. For the operator $e^{\frac{1}{2} \tau T}$, it can be discretized in space via Fourier spectral method and then integrate (in phase space or Fourier space) in time exactly. For details, we refer to [9]13] and references therein. In fact, the implementation of the $S_{4 \mathrm{c}}$ method in this case is much simpler than that of the $S_{4}$ and $S_{4 \mathrm{RK}}$ methods.

Of course, when the magnetic potential is nonzero in the Dirac equation (1.17) in 2D and (1.7) in 2D and 3D, one has to adapt the formulation (4.20) for $S_{4 \mathrm{c}}$ method. In this case, the main difficulty is how to efficiently and accurately evaluate the operator $e^{\frac{2}{3} \tau \hat{W}}$. This can be done by using the method of characteristics and the nonuniform fast Fourier transform (NUFFT), which has been developed for the magnetic Schrödinger equation. For details, we refer to [19,44] and references therein. Of course, it is a little more tedious in practical implementation for $S_{4 \mathrm{c}}$ method than that for the $S_{4}$ and $S_{4 \mathrm{RK}}$ methods in this situation.

\section{Comparision of different time-splitting methods}

In this section, we compare the fourth-order compact time-splitting Fourier pseudospectral $S_{4 \mathrm{c}}$ method 4.11) with other time-splitting methods including the first-order time-splitting $\left(S_{1}\right)$ method, the second-order time-splitting $\left(S_{2}\right)$ method, the fourth-order time-splitting $\left(S_{4}\right)$ method and the fourth-order partitioned Runge-Kutta time-splitting $\left(S_{4 \mathrm{RK}}\right)$ method in terms of accuracy and efficiency as well as long time behavior.

\subsection{An example in 1D}

For simplicity, we first consider an example in 1D. In the Dirac equation 1.17, we take $d=1, \varepsilon=\delta=v=1$ and

$$
V(x)=\frac{1-x}{1+x^{2}}, \quad A_{1}(x)=\frac{(x+1)^{2}}{1+x^{2}}, \quad x \in \mathbb{R} .
$$

The initial data in 1.18 is taken as:

$$
\phi_{1}(0, x)=e^{-x^{2} / 2}, \quad \phi_{2}(0, x)=e^{-(x-1)^{2} / 2}, \quad x \in \mathbb{R} .
$$

The problem is solved numerically on a bounded domain $\Omega=(-32,32)$, i.e. $a=-32$ and $b=32$. 


\begin{tabular}{|c|cccc|}
\hline & $h_{0}=1$ & $h_{0} / 2$ & $h_{0} / 2^{2}$ & $h_{0} / 2^{3}$ \\
\hline$S_{1}$ & 1.01 & $5.16 \mathrm{E}-2$ & $7.07 \mathrm{E}-5$ & - \\
\hline$S_{2}$ & 1.01 & $5.16 \mathrm{E}-2$ & $6.96 \mathrm{E}-5$ & $1.92 \mathrm{E}-10$ \\
\hline$S_{4}$ & 1.01 & $5.16 \mathrm{E}-2$ & $6.96 \mathrm{E}-5$ & $3.52 \mathrm{E}-10$ \\
\hline$S_{4 \mathrm{c}}$ & 1.01 & $5.16 \mathrm{E}-2$ & $6.96 \mathrm{E}-5$ & $3.06 \mathrm{E}-10$ \\
\hline$S_{4 \mathrm{RK}}$ & 1.01 & $5.16 \mathrm{E}-2$ & $6.96 \mathrm{E}-5$ & $5.15 \mathrm{E}-10$ \\
\hline
\end{tabular}

Table 5.1 Spatial errors $e_{\Phi}(t=6)$ of different time-splitting methods under different mesh size $h$ for the Dirac equation 1.17 in 1D.

Due to the fact that the exact solution is not available, we obtain a numerical 'exact' solution by using the $S_{4 \mathrm{c}}$ method with a fine mesh size $h_{e}=\frac{1}{16}$ and a small time step $\tau_{e}=$ $10^{-5}$. Let $\Phi^{n}$ be the numerical solution obtained by a numerical method with mesh size $h$ and time step $\tau$. Then the error is quantified as

$$
e_{\Phi}\left(t_{n}\right)=\left\|\Phi^{n}-\Phi\left(t_{n}, \cdot\right)\right\|_{l^{2}}=\sqrt{h \sum_{j=0}^{M-1}\left|\Phi\left(t_{n}, x_{j}\right)-\Phi_{j}^{n}\right|^{2}} .
$$

In order to compare the spatial errors, we take time step $\tau=\tau_{e}=10^{-5}$ such that the temporal discretization error could be negligible. Table 5.1 lists numerical errors $e_{\Phi}(t=6)$ for different time-splitting methods under different mesh size $h$. We remark here that, for the $S_{1}$ method, in order to observe the spatial error when the mesh size $h=h_{0} / 2^{3}$, one has to choose time step $\tau \leq 10^{-10}$ which is too small and thus the error is not shown in the table for this case. From Table 5.1 we could see that all the numerical methods are spectral order accurate in space (cf. each row in Table 5.1).

In order to compare the temporal errors, we take mesh size $h=h_{e}=\frac{1}{16}$ such that the spatial discretization error could be negligible. Table 5.2 lists numerical errors $e_{\Phi}(t=6)$ for different time-splitting methods under different time step $\tau$. In the table, we use second (s) as the unit for CPU time. For comparison, Figure 5.1 plots $e_{\Phi}(t=6)$ and $e_{\Phi}(t=6) / \tau^{\alpha}$ with $\alpha$ taken as the order of accuracy of a certain numerical method (in order to show the constants $C_{1}$ in (2.6), $C_{2}$ in (2.8), $C_{4}$ in (2.11), $\widetilde{C}_{4}$ in (2.13) and $\widehat{C}_{4}$ in (2.16) for different time-splitting methods under different time step $\tau$.

From Table 5.2 and Figure 5.1, we can draw the following conclusions: (i) The $S_{1}$ method is first-order in time, the $S_{2}$ method is second-order in time, and the $S_{4}, S_{4 \mathrm{c}}$ and $S_{4 \mathrm{RK}}$ methods are all fourth-order in time (cf. Table 5.2 and Figure 5.1 left). (ii) For any fixed mesh $h$ and time $\tau$, the computational time for $S_{1}$ and $S_{2}$ are quite similar, the computational time of $S_{4 \mathrm{c}}, S_{4}$ and $S_{4 \mathrm{RK}}$ are about two times, three times and six times of the $S_{2}$ method, respectively (cf. Table 5.2). (iii) Among the three fourth-order time-splitting methods, $S_{4 \mathrm{c}}$ and $S_{4 \mathrm{RK}}$ are quite similar in terms of numerical errors for any fixed $\tau$ and they are much smaller than that of the $S_{4}$ method, especially when the $\tau$ is not so small (cf. Table 


\begin{tabular}{|c|c|ccccccc|}
\hline \multicolumn{2}{|c|}{} & $\tau_{0}=1 / 2$ & $\tau_{0} / 2$ & $\tau_{0} / 2^{2}$ & $\tau_{0} / 2^{3}$ & $\tau_{0} / 2^{4}$ & $\tau_{0} / 2^{5}$ & $\tau_{0} / 2^{6}$ \\
\hline \multirow{3}{*}{$S_{1}$} & $e_{\Phi}(t=6)$ & 1.17 & $4.71 \mathrm{E}-1$ & $2.09 \mathrm{E}-1$ & $9.90 \mathrm{E}-2$ & $4.82 \mathrm{E}-2$ & $2.38 \mathrm{E}-2$ & $1.18 \mathrm{E}-2$ \\
& rate & - & 1.31 & 1.17 & 1.08 & 1.04 & 1.02 & 1.01 \\
& CPU Time & 0.02 & 0.05 & 0.11 & 0.16 & 0.37 & 0.62 & $\mathbf{1 . 3 1}$ \\
\hline \multirow{5}{*}{$S_{2}$} & $e_{\Phi}(t=6)$ & $7.49 \mathrm{E}-1$ & $1.87 \mathrm{E}-1$ & $4.66 \mathrm{E}-2$ & $1.16 \mathrm{E}-2$ & $2.91 \mathrm{E}-3$ & $7.27 \mathrm{E}-4$ & $1.82 \mathrm{E}-4$ \\
& rate & - & 2.00 & 2.00 & 2.00 & 2.00 & 2.00 & 2.00 \\
& CPU Time & 0.04 & 0.06 & 0.11 & 0.21 & 0.37 & 0.75 & $\mathbf{1 . 4 2}$ \\
\hline \multirow{3}{*}{$S_{4}$} & $e_{\Phi}(t=6)$ & $3.30 \mathrm{E}-1$ & $3.73 \mathrm{E}-2$ & $3.05 \mathrm{E}-3$ & $2.07 \mathrm{E}-4$ & $1.32 \mathrm{E}-5$ & $8.29 \mathrm{E}-7$ & $5.20 \mathrm{E}-8$ \\
& rate & - & 3.15 & 3.61 & 3.89 & 3.97 & 3.99 & 4.00 \\
& CPU Time & 0.10 & 0.16 & 0.38 & 0.58 & 1.09 & 2.23 & $\mathbf{4 . 4 1}$ \\
\hline \multirow{3}{*}{$S_{4 \mathrm{c}}$} & $e_{\Phi}(t=6)$ & $1.66 \mathrm{E}-2$ & $9.54 \mathrm{E}-4$ & $5.90 \mathrm{E}-5$ & $3.68 \mathrm{E}-6$ & $2.30 \mathrm{E}-7$ & $1.43 \mathrm{E}-8$ & $8.12 \mathrm{E}-10$ \\
& rate & - & 4.12 & 4.01 & 4.00 & 4.00 & 4.01 & 4.13 \\
& CPU Time & 0.06 & 0.09 & 0.18 & 0.35 & 0.68 & 1.36 & $\mathbf{2 . 6 8}$ \\
\hline \multirow{3}{*}{$S_{4 \mathrm{RK}}$} & rate & - & 4.01 & 3.99 & 4.00 & 4.00 & 4.07 & 3.96 \\
& CPU Time & 0.15 & 0.28 & 0.57 & 1.24 & 2.66 & 3.94 & $\mathbf{7 . 7 9}$ \\
\hline
\end{tabular}

Table 5.2 Temporal errors $e_{\Phi}(t=6)$ of different time-splitting methods under different time step $\tau$ for the Dirac equation 1.17 in 1D. Here we also list convergence rates and computational time (CPU time in seconds) for comparison.
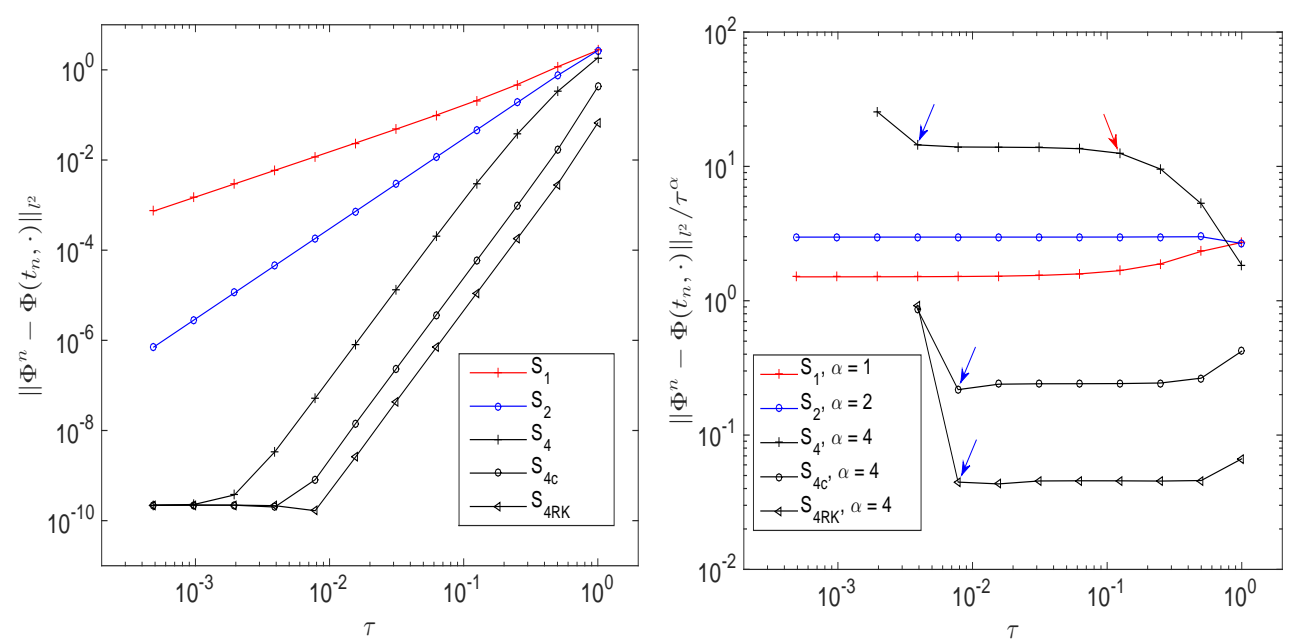

Fig. 5.1 Temporal errors $e_{\Phi}(t=6)$ (left) and $e_{\Phi}(t=6) / \tau^{\alpha}$ with $\alpha$ taken as the order of accuracy of a certain numerical method (right) of different time-splitting methods under different time step $\tau$ for the Dirac equation 1.17 in $1 \mathrm{D}$

5.2 and Figure 5.1 left). (iv) For the constants in front of the convergence rates of different methods, $C_{4} \gg C_{1} \sim C_{2} \gg \widehat{C_{4}} \sim \widetilde{C}_{4}$ (cf. Figure 5.1 right). (v) For the $S_{4}$ method, it suffers 


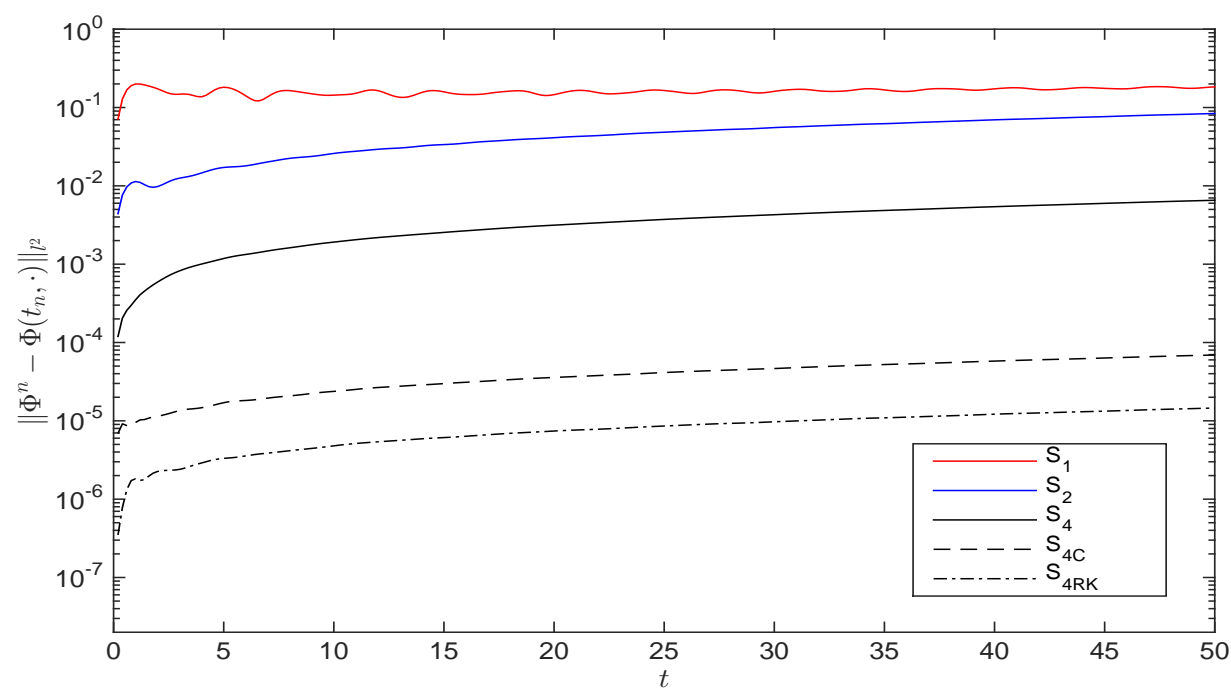

Fig. 5.2 Time evolution of the errors $e_{\Phi}(t)$ under $h=\frac{1}{16}$ and $\tau=0.1$ over long time of different time-splitting methods for the Dirac equation 1.17 in 1D.

from convergence rate reduction when the time step is not small and a very large constant in front of the convergence rate. Thus this method is, in general, to be avoided in practical computation, which has been observed when it is applied for the nonlinear Schrödinger equation too [59].

To compare the long time behavior of different time-splitting methods, Figure 5.2 depicts $e_{\Phi}(t)$ under mesh size $h=\frac{1}{16}$ and time step $\tau=0.1$ for $0 \leq t \leq T:=50$.

From Figure 5.2 we can observe: (i) The errors increase very fast when $t$ is small, e.g. $0 \leq t \leq O(1)$, and they almost don't change when $t \gg 1$, thus they are suitable for long time simulation, especially the fourth-order methods. (ii) When $t$ is not large, the error of the $S_{4}$ method is about 10 times bigger than that of the $S_{4 \mathrm{c}}$ method; however, when $t \gg 1$, it becomes about 100 times larger. (iii) The error of the $S_{4 \mathrm{RK}}$ method is always the smallest among all the time-splitting methods.

Based on the efficiency and accuracy as well as long time behavior, in conclusion, for the three fourth-order time-splitting methods, $S_{4 \mathrm{c}}$ is more accurate than $S_{4}$ and it is more efficient than $S_{4 \mathrm{RK}}$. Thus the $S_{4 \mathrm{c}}$ method is highly recommended for studying the dynamics of the Dirac equation, especially in $1 \mathrm{D}$.

\subsection{An example in 2D}

For simplicity, here we only compare the three fourth-order integrators, i.e., $S_{4 \mathrm{c}}, S_{4}$ and $S_{4 \mathrm{RK}}$ via an example in 2D. In order to do so, in the Dirac equation (1.17), we take $d=2$, 


\begin{tabular}{|c|cccc|}
\hline & $h_{0}=1 / 2$ & $h_{0} / 2$ & $h_{0} / 2^{2}$ & $h_{0} / 2^{3}$ \\
\hline$S_{4}$ & 1.10 & $1.01 \mathrm{E}-1$ & $3.83 \mathrm{E}-4$ & $7.33 \mathrm{E}-10$ \\
\hline$S_{4 \mathrm{c}}$ & 1.10 & $1.01 \mathrm{E}-1$ & $3.83 \mathrm{E}-4$ & $7.33 \mathrm{E}-10$ \\
\hline$S_{4 \mathrm{RK}}$ & 1.10 & $1.01 \mathrm{E}-1$ & $3.83 \mathrm{E}-4$ & $7.34 \mathrm{E}-10$ \\
\hline
\end{tabular}

Table 5.3 Spatial errors $e_{\Phi}(t=2)$ of different time-splitting methods under different mesh size $h$ for the Dirac equation 1.17 in $2 \mathrm{D}$.

$\varepsilon=\delta=v=1$ and take the potential in honey-comb form

$$
\begin{aligned}
& V(\mathbf{x})=\cos \left(\frac{4 \pi}{\sqrt{3}} \mathbf{e}_{\mathbf{1}} \cdot \mathbf{x}\right)+\cos \left(\frac{4 \pi}{\sqrt{3}} \mathbf{e}_{2} \cdot \mathbf{x}\right)+\cos \left(\frac{4 \pi}{\sqrt{3}} \mathbf{e}_{3} \cdot \mathbf{x}\right), \\
& A_{1}(\mathbf{x})=A_{2}(\mathbf{x})=0, \quad \mathbf{x} \in \mathbb{R}^{2}
\end{aligned}
$$

with

$$
\mathbf{e}_{1}=(-1,0)^{T}, \quad \mathbf{e}_{2}=(1 / 2, \sqrt{3} / 2)^{T}, \quad \mathbf{e}_{3}=(1 / 2,-\sqrt{3} / 2)^{T} .
$$

The initial data in (1.18) is taken as:

$$
\phi_{1}(0, \mathbf{x})=e^{-\frac{x^{2}+y^{2}}{2}}, \quad \phi_{2}(0, \mathbf{x})=e^{-\frac{(x-1)^{2}+y^{2}}{2}}, \quad \mathbf{x}=(x, y)^{T} \in \mathbb{R}^{2} .
$$

The problem is solved numerically on a bounded domain $\Omega=(-10,10) \times(-10,10)$.

Similar to the 1D example, we obtain a numerical 'exact' solution by using the $S_{4 \mathrm{c}}$ method with a fine mesh size $h_{e}=\frac{1}{32}$ and a small time step $\tau_{e}=10^{-4}$. The error for the numerical solution $\Phi^{n}$ with mesh size $h$ and time step $\tau$ is quantified as

$$
e_{\Phi}\left(t_{n}\right)=\left\|\Phi^{n}-\Phi\left(t_{n}, \cdot\right)\right\|_{l^{2}}=h \sqrt{\sum_{j=0}^{M-1} \sum_{l=0}^{M-1}\left|\Phi\left(t_{n}, x_{j}, y_{l}\right)-\Phi_{j l}^{n}\right|^{2}}
$$

Similar to the 1D case, in order to compare the spatial errors, we take time step $\tau=$ $\tau_{e}=10^{-4}$ such that the temporal discretization error could be negligible. Table 5.3 lists numerical errors $e_{\Phi}(t=2)$ for different time-splitting methods under different mesh size $h$. In order to compare the temporal errors, we take mesh size $h=h_{e}=\frac{1}{32}$ such that the spatial discretization error could be negligible. Table 5.4 lists numerical errors $e_{\Phi}(t=2)$ for different time-splitting methods under different time step $\tau$.

From Tables 5.3 8.4 , we can draw the following conclusions: (i) All the three methods are spectrally accurate in space and fourth-order in time. (ii) For any fixed mesh size $h$ and time step $\tau$, the computational times of the $S_{4}$ and $S_{4 \mathrm{RK}}$ methods are approximately 1.5 times and 3 times more than that of the $S_{4 \mathrm{c}}$ method, respectively. (iii) $S_{4 \mathrm{c}}$ and $S_{4 \mathrm{RK}}$ are quite similar in terms of numerical errors for any fixed $\tau$ and the errors are much smaller than that of the $S_{4}$ method, especially when $\tau$ is not so small. (iv) Again, order reduction in time was 


\begin{tabular}{|c|c|ccccccc|}
\hline \multicolumn{2}{|c|}{} & $\tau_{0}=1 / 2$ & $\tau_{0} / 2$ & $\tau_{0} / 2^{2}$ & $\tau_{0} / 2^{3}$ & $\tau_{0} / 2^{4}$ & $\tau_{0} / 2^{5}$ & $\tau_{0} / 2^{6}$ \\
\hline \multirow{3}{*}{$S_{4}$} & Error & $4.33 \mathrm{E}-1$ & $2.57 \mathrm{E}-2$ & $3.53 \mathrm{E}-3$ & $2.83 \mathrm{E}-4$ & $1.88 \mathrm{E}-5$ & $1.20 \mathrm{E}-6$ & $7.51 \mathrm{E}-8$ \\
& Order & - & 4.07 & 2.87 & 3.64 & 3.91 & 3.98 & 3.99 \\
& CPU Time & 0.20 & 0.26 & 0.45 & 1.04 & 1.63 & 3.37 & $\mathbf{6 . 5 4}$ \\
\hline \multirow{5}{*}{$S_{4 c}$} & Error & $6.75 \mathrm{E}-2$ & $3.18 \mathrm{E}-3$ & $7.91 \mathrm{E}-5$ & $4.70 \mathrm{E}-6$ & $2.91 \mathrm{E}-7$ & $1.81 \mathrm{E}-8$ & $1.13 \mathrm{E}-9$ \\
& Order & - & 4.41 & 5.33 & 4.07 & 4.01 & 4.00 & 4.00 \\
& CPU Time & 0.12 & 0.28 & 0.31 & 0.55 & 1.11 & 2.09 & $\mathbf{4 . 1 4}$ \\
\hline \multirow{3}{*}{$S_{4 \mathrm{RK}}$} & Error & $8.32 \mathrm{E}-3$ & $3.56 \mathrm{E}-4$ & $7.42 \mathrm{E}-6$ & $4.43 \mathrm{E}-7$ & $2.75 \mathrm{E}-8$ & $1.71 \mathrm{E}-9$ & $1.07 \mathrm{E}-10$ \\
& Order & - & 4.55 & 5.59 & 4.07 & 4.01 & 4.00 & 4.00 \\
& CPU Time & 0.26 & 0.43 & 0.87 & 1.52 & 2.92 & 6.20 & $\mathbf{1 1 . 7 4}$ \\
\hline
\end{tabular}

Table 5.4 Temporal errors $e_{\Phi}(t=2)$ of different fourth order time-splitting methods under different time step $\tau$ for the Dirac equation (1.17) in 2D. Here we also list convergence rates and computational time (CPU time in seconds) for comparison.

observed in the $S_{4}$ method when $\tau$ is not small, however, there is almost no order reduction in time for the $S_{4 \mathrm{c}}$ and $S_{4 \mathrm{RK}}$ methods.

Again, based on the efficiency and accuracy for the Dirac equation in high dimensions, in conclusion, for the three fourth-order time-splitting methods, $S_{4 \mathrm{c}}$ is more accurate than $S_{4}$ and it is more efficient than $S_{4 \mathrm{RK}}$. Thus the $S_{4 \mathrm{c}}$ method is highly recommended for studying the dynamics of the Dirac equation in high dimensions, especially without magnetic potential.

\section{Spatial/temporal resolution of the $S_{4 \mathrm{c}}$ method in different parameter regimes}

In this section, we study numerically temproal/spatial resolution of the fourth-order compact time-splitting Fourier pseudospectral $S_{4 \mathrm{c}}$ method 4.11 for the Dirac equation in different parameter regimes. We take $d=1$ and the electromagnetic potentials as $(5.1)$ in Dirac equation 1.17. To quantify the numerical error, we adapt the relative errors of the wave function $\Phi$, the total probability density $\rho$ and the current $\mathbf{J}$ as

$$
e_{\Phi}^{r}\left(t_{n}\right)=\frac{\left\|\Phi^{n}-\Phi\left(t_{n}, \cdot\right)\right\|_{l^{2}}}{\left\|\Phi\left(t_{n}, \cdot\right)\right\|_{l^{2}}}, \quad e_{\rho}^{r}\left(t_{n}\right)=\frac{\left\|\rho^{n}-\rho\left(t_{n}, \cdot\right)\right\|_{l^{2}}}{\left\|\rho\left(t_{n}, \cdot\right)\right\|_{l^{2}}}, \quad e_{\mathbf{J}}^{r}\left(t_{n}\right)=\frac{\left\|\mathbf{J}^{n}-\mathbf{J}\left(t_{n}, \cdot\right)\right\|_{l^{2}}}{\left\|\mathbf{J}\left(t_{n}, \cdot\right)\right\|_{l^{2}}},
$$

where $\rho^{n}$ and $\mathbf{J}^{n}$ are obtained from the wave function $\Phi^{n}$ via (1.21) and (1.23) with $d=1$, respectively. Again, the numerical 'exact' solution is obtained by using the $S_{4 \mathrm{c}}$ method with a very fine mesh $h=h_{e}$ and a very small time step $\tau=\tau_{e}$. 


\begin{tabular}{ccccccc}
\hline & $\tau_{0}=1$ & $\tau_{0} / 2^{2}$ & $\tau_{0} / 2^{4}$ & $\tau_{0} / 2^{6}$ & $\tau_{0} / 2^{8}$ & $\tau_{0} / 2^{10}$ \\
\hline$\varepsilon_{0}=1$ & $2.24 \mathrm{E}-1$ & $\mathbf{5 . 0 7 E - 4}$ & $1.95 \mathrm{E}-6$ & $7.63 \mathrm{E}-9$ & $<1 \mathrm{E}-10$ & $<1 \mathrm{E}-10$ \\
order & - & $\mathbf{4 . 3 9}$ & 4.01 & 4.00 & - & - \\
\hline$\varepsilon_{0} / 2$ & 1.18 & $1.05 \mathrm{E}-2$ & $\mathbf{3 . 6 1 E - 5}$ & $1.40 \mathrm{E}-7$ & $5.67 \mathrm{E}-10$ & $<1 \mathrm{E}-10$ \\
order & - & 3.41 & $\mathbf{4 . 0 9}$ & 4.00 & 3.97 & - \\
\hline$\varepsilon_{0} / 2^{2}$ & 1.46 & $2.07 \mathrm{E}-1$ & $1.69 \mathrm{E}-3$ & $\mathbf{6 . 0 9 E}-\mathbf{6}$ & $2.37 \mathrm{E}-8$ & $<1 \mathrm{E}-10$ \\
order & - & 1.41 & 3.47 & $\mathbf{4 . 0 6}$ & 4.00 & - \\
\hline$\varepsilon_{0} / 2^{3}$ & 1.41 & 1.50 & $5.88 \mathrm{E}-2$ & $3.84 \mathrm{E}-4$ & $\mathbf{1 . 3 9 E}-\mathbf{6}$ & $5.40 \mathrm{E}-9$ \\
order & - & -0.04 & 2.33 & 3.63 & $\mathbf{4 . 0 6}$ & 4.00 \\
\hline$\varepsilon_{0} / 2^{4}$ & 1.43 & 1.47 & $6.80 \mathrm{E}-1$ & $1.46 \mathrm{E}-2$ & $9.33 \mathrm{E}-5$ & $\mathbf{3 . 3 8 E}-7$ \\
order & - & -0.02 & 0.56 & 2.77 & 3.65 & $\mathbf{4 . 0 5}$ \\
\hline
\end{tabular}

Table 6.1 Temporal errors $e_{\Phi}^{r}(t=6)$ of $S_{4 \mathrm{c}}$ under different $\tau$ and $\varepsilon$ for the Dirac equation 1.17 in 1D in the nonrelativistic limit regime.

6.1 In the nonrelativistic limit regime

Here we take $\delta=v=1, \varepsilon \in(0,1]$ and the initial data in (1.18) is taken as (5.2). In this parameter regime, the solution propagates waves with wavelength at $O(1)$ and $O\left(\varepsilon^{2}\right)$ in space and time, respectively. The problem is solved numerically on a bounded domain $\Omega=(-32,32)$, i.e. $a=-32$ and $b=32$. Similar to the second-order time-splitting Fourier pseudospectral method [9], the $S_{4 \mathrm{c}}$ method converges uniformly with respect to $\varepsilon \in(0,1]$ at spectral order in space. Detailed numerical results are omitted here for brevity. Here we only present temporal errors by taking $h=h_{e}=\frac{1}{16}$ so that the spatial discretization error could be negligible. Table 6.1 shows the temporal errors $e_{\Phi}^{r}(t=6)$ for the wave function under different $\tau$ and $\varepsilon \in(0,1]$. Similarly, Tables 6.2 and 6.3 depict the temporal errors $e_{\rho}^{r}(t=6)$ and $e_{\mathbf{J}}^{r}(t=6)$ for the probability and current, respectively.

From Tables 6.16.3, when $\tau \lesssim \varepsilon^{2}$, fourth-order convergence is observed for the $S_{4 \mathrm{c}}$ method in the relative error for the wave function, probability and current. This suggests that the $\varepsilon$-scalability for the $S_{4 \mathrm{c}}$ method in the nonrelativistic limit regime is: $h=O(1)$ and $\tau=O\left(\varepsilon^{2}\right)$. In addition, noticing $\Phi=O(1), \rho=O(1)$ and $\mathbf{J}=O\left(\varepsilon^{-1}\right)$ when $0 \leq \varepsilon \ll 1$, we can formally observe the following error bounds for $0<\varepsilon \leq 1, \tau \lesssim \varepsilon^{2}$ and $0 \leq n \leq \frac{T}{\tau}$

$$
\begin{aligned}
& \left\|\Phi^{n}-\Phi\left(t_{n}, \cdot\right)\right\|_{l^{2}} \lesssim h^{m_{0}}+\frac{\tau^{4}}{\varepsilon^{6}}, \quad\left\|\rho^{n}-\rho\left(t_{n}, \cdot\right)\right\|_{l^{2}} \lesssim h^{m_{0}}+\frac{\tau^{4}}{\varepsilon^{6}}, \\
& \left\|\mathbf{J}^{n}-\mathbf{J}\left(t_{n}, \cdot\right)\right\|_{l^{2}} \lesssim \frac{1}{\varepsilon}\left(h^{m_{0}}+\frac{\tau^{4}}{\varepsilon^{6}}\right) .
\end{aligned}
$$

where $m_{0} \geq 2$ depends on the regularity of the solution. Rigorous mathematical justification is still on-going. 


\begin{tabular}{ccccccc}
\hline & $\tau_{0}=1$ & $\tau_{0} / 2^{2}$ & $\tau_{0} / 2^{4}$ & $\tau_{0} / 2^{6}$ & $\tau_{0} / 2^{8}$ & $\tau_{0} / 2^{10}$ \\
\hline$\varepsilon_{0}=1$ & $1.71 \mathrm{E}-1$ & $\mathbf{3 . 7 3 E}-4$ & $1.44 \mathrm{E}-6$ & $5.62 \mathrm{E}-9$ & $<1 \mathrm{E}-10$ & $<1 \mathrm{E}-10$ \\
order & - & $\mathbf{4 . 4 2}$ & 4.01 & 4.00 & - & - \\
\hline$\varepsilon_{0} / 2$ & 1.31 & $7.17 \mathrm{E}-3$ & $\mathbf{2 . 4 5 E - 5}$ & $9.50 \mathrm{E}-8$ & $3.94 \mathrm{E}-10$ & $<1 \mathrm{E}-10$ \\
order & - & 3.76 & $\mathbf{4 . 1 0}$ & 4.01 & 3.96 & - \\
\hline$\varepsilon_{0} / 2^{2}$ & $8.19 \mathrm{E}-1$ & $2.20 \mathrm{E}-1$ & $8.16 \mathrm{E}-4$ & $\mathbf{2 . 9 2 E}-\mathbf{6}$ & $1.13 \mathrm{E}-8$ & $<1 \mathrm{E}-10$ \\
order & - & 0.95 & 4.04 & $\mathbf{4 . 0 6}$ & 4.00 & - \\
\hline$\varepsilon_{0} / 2^{3}$ & $8.75 \mathrm{E}-1$ & $4.77 \mathrm{E}-1$ & $5.76 \mathrm{E}-2$ & $1.65 \mathrm{E}-4$ & $\mathbf{5 . 8 9 E}-\mathbf{7}$ & $2.29 \mathrm{E}-9$ \\
order & - & 0.44 & 1.52 & 4.22 & $\mathbf{4 . 0 7}$ & 4.00 \\
\hline$\varepsilon_{0} / 2^{4}$ & 1.00 & 1.12 & $2.04 \mathrm{E}-1$ & $1.49 \mathrm{E}-2$ & $4.03 \mathrm{E}-5$ & $\mathbf{1 . 4 3 E - 7}$ \\
order & - & -0.08 & 1.23 & 1.88 & 4.27 & $\mathbf{4 . 0 7}$ \\
\hline
\end{tabular}

Table 6.2 Temporal errors $e_{\rho}^{r}(t=6)$ of $S_{4 \mathrm{c}}$ under different $\tau$ and $\varepsilon$ for the Dirac equation (1.17) in 1D in the nonrelativistic limit regime.

\begin{tabular}{ccccccc}
\hline & $\tau_{0}=1$ & $\tau_{0} / 2^{2}$ & $\tau_{0} / 2^{4}$ & $\tau_{0} / 2^{6}$ & $\tau_{0} / 2^{8}$ & $\tau_{0} / 2^{10}$ \\
\hline$\varepsilon_{0}=1$ & $2.92 \mathrm{E}-1$ & $\mathbf{6 . 7 6 E}-4$ & $2.61 \mathrm{E}-6$ & $1.02 \mathrm{E}-8$ & $<1 \mathrm{E}-10$ & $<1 \mathrm{E}-10$ \\
order & - & $\mathbf{4 . 3 8}$ & 4.01 & 4.00 & - & - \\
\hline$\varepsilon_{0} / 2$ & 1.30 & $1.98 \mathrm{E}-2$ & $\mathbf{6 . 8 8 E}-5$ & $2.67 \mathrm{E}-7$ & $1.06 \mathrm{E}-9$ & $<1 \mathrm{E}-10$ \\
order & - & 3.02 & $\mathbf{4 . 0 9}$ & 4.00 & 3.99 & - \\
\hline$\varepsilon_{0} / 2^{2}$ & 1.29 & $2.98 \mathrm{E}-1$ & $3.40 \mathrm{E}-3$ & $\mathbf{1 . 2 3 E}-\mathbf{5}$ & $4.76 \mathrm{E}-8$ & $<1 \mathrm{E}-10$ \\
order & - & 1.06 & 3.23 & $\mathbf{4 . 0 6}$ & 4.00 & - \\
\hline$\varepsilon_{0} / 2^{3}$ & 1.21 & 1.29 & $8.82 \mathrm{E}-2$ & $7.85 \mathrm{E}-4$ & $\mathbf{2 . 8 5 E}-\mathbf{6}$ & $1.11 \mathrm{E}-8$ \\
order & - & -0.05 & 1.94 & 3.41 & $\mathbf{4 . 0 5}$ & 4.00 \\
\hline$\varepsilon_{0} / 2^{4}$ & 1.52 & 1.44 & 1.30 & $2.41 \mathrm{E}-2$ & $1.92 \mathrm{E}-4$ & $\mathbf{6 . 9 8 E}-\mathbf{7}$ \\
order & - & 0.04 & 0.07 & 2.88 & 3.48 & $\mathbf{4 . 0 5}$ \\
\hline
\end{tabular}

Table 6.3 Temporal errors $e_{\mathbf{J}}^{r}(t=6)$ of $S_{4 \mathrm{c}}$ under different $\tau$ and $\varepsilon$ for the Dirac equation 1.17 in 1D in the nonrelativistic limit regime.

6.2 In the semiclassical limit regime

Here we take $\varepsilon=v=1, \delta \in(0,1]$. The initial data in 1.18$)$ is taken as

$$
\begin{aligned}
& \phi_{1}(0, x)=\frac{1}{2} e^{-4 x^{2}} e^{i S_{0}(x) / \delta}\left(1+\sqrt{1+S_{0}^{\prime}(x)^{2}}\right) \\
& \phi_{2}(0, x)=\frac{1}{2} e^{-4 x^{2}} e^{i S_{0}(x) / \delta} S_{0}^{\prime}(x), \quad x \in \mathbb{R}
\end{aligned}
$$

with

$$
S_{0}(x)=\frac{1}{40}(1+\cos (2 \pi x)), \quad x \in \mathbb{R}
$$




\begin{tabular}{cccccccc}
\hline & $h_{0}=1$ & $h_{0} / 2$ & $h_{0} / 2^{2}$ & $h_{0} / 2^{3}$ & $h_{0} / 2^{4}$ & $h_{0} / 2^{5}$ & $h_{0} / 2^{6}$ \\
\hline$\delta_{0}=1$ & $\mathbf{8 . 2 5 E}-1$ & $2.00 \mathrm{E}-1$ & $9.52 \mathrm{E}-3$ & $6.66 \mathrm{E}-6$ & $3.78 \mathrm{E}-10$ & $<1 \mathrm{E}-10$ & $<1 \mathrm{E}-10$ \\
\hline$\delta_{0} / 2$ & 1.20 & $\mathbf{7 . 4 0 E}-1$ & $5.31 \mathrm{E}-2$ & $8.87 \mathrm{E}-5$ & $3.43 \mathrm{E}-10$ & $<1 \mathrm{E}-10$ & $<1 \mathrm{E}-10$ \\
\hline$\delta_{0} / 2^{2}$ & 1.41 & $9.89 \mathrm{E}-1$ & $\mathbf{5 . 1 2 E}-1$ & $3.81 \mathrm{E}-3$ & $9.24 \mathrm{E}-10$ & $<1 \mathrm{E}-10$ & $<1 \mathrm{E}-10$ \\
\hline$\delta_{0} / 2^{3}$ & 1.76 & 1.21 & $7.30 \mathrm{E}-1$ & $\mathbf{2 . 7 6 E}-1$ & $1.91 \mathrm{E}-5$ & $4.17 \mathrm{E}-10$ & $<1 \mathrm{E}-10$ \\
\hline$\delta_{0} / 2^{4}$ & 1.37 & 1.36 & 1.36 & $5.31 \mathrm{E}-1$ & $\mathbf{1 . 5 4 E}-1$ & $5.31 \mathrm{E}-10$ & $<1 \mathrm{E}-10$ \\
\hline$\delta_{0} / 2^{5}$ & 2.44 & 1.92 & 1.36 & 1.36 & $4.36 \mathrm{E}-1$ & $\mathbf{5 . 4 9 E}-2$ & $2.90 \mathrm{E}-10$ \\
\hline
\end{tabular}

Table 6.4 Spatial errors $e_{\Phi}^{r}(t=2)$ of $S_{4 \mathrm{c}}$ under different $h$ and $\delta$ for the Dirac equation 1.17 in 1D in the semiclassical limit regime.

\begin{tabular}{cccccccc}
\hline & $h_{0}=1$ & $h_{0} / 2$ & $h_{0} / 2^{2}$ & $h_{0} / 2^{3}$ & $h_{0} / 2^{4}$ & $h_{0} / 2^{5}$ & $h_{0} / 2^{6}$ \\
\hline$\delta_{0}=1$ & $\mathbf{5 . 8 3 E}-1$ & $1.39 \mathrm{E}-1$ & $8.27 \mathrm{E}-3$ & $4.36 \mathrm{E}-6$ & $4.92 \mathrm{E}-10$ & $<1 \mathrm{E}-10$ & $<1 \mathrm{E}-10$ \\
\hline$\delta_{0} / 2$ & 1.29 & $\mathbf{5 . 2 2 E}-1$ & $3.71 \mathrm{E}-2$ & $5.56 \mathrm{E}-5$ & $2.79 \mathrm{E}-10$ & $<1 \mathrm{E}-10$ & $<1 \mathrm{E}-10$ \\
\hline$\delta_{0} / 2^{2}$ & $9.22 \mathrm{E}-1$ & $7.44 \mathrm{E}-1$ & $\mathbf{2 . 4 1 E}-1$ & $1.54 \mathrm{E}-3$ & $6.75 \mathrm{E}-10$ & $<1 \mathrm{E}-10$ & $<1 \mathrm{E}-10$ \\
\hline$\delta_{0} / 2^{3}$ & 1.63 & $9.39 \mathrm{E}-1$ & $6.11 \mathrm{E}-1$ & $\mathbf{6 . 3 3 E}-2$ & $4.78 \mathrm{E}-6$ & $8.19 \mathrm{E}-10$ & $<1 \mathrm{E}-10$ \\
\hline$\delta_{0} / 2^{4}$ & 2.04 & 1.40 & 1.00 & $3.57 \mathrm{E}-1$ & $\mathbf{1 . 9 7 E}-2$ & $6.76 \mathrm{E}-10$ & $<1 \mathrm{E}-10$ \\
\hline$\delta_{0} / 2^{5}$ & 5.81 & 3.65 & 1.07 & 1.01 & $1.86 \mathrm{E}-1$ & $\mathbf{3 . 3 5 E}-3$ & $5.67 \mathrm{E}-10$ \\
\hline
\end{tabular}

Table 6.5 Spatial errors $e_{\rho}^{r}(t=2)$ of $S_{4 \mathrm{c}}$ under different $h$ and $\delta$ for the Dirac equation 1.17 in 1D in the semiclassical limit regime.

In this parameter regime, the solution propagates waves with wavelength at $O(\delta)$ in both space and time. The problem is solved numerically on a bounded domain $\Omega=(-16,16)$, i.e. $a=-16$ and $b=16$.

Table 6.4 shows the spatial errors $e_{\Phi}^{r}(t=2)$ for the wave function under different $h$ and $\delta \in(0,1]$ with $\tau=\tau_{e}=10^{-4}$ such that the temporal discretization error could be negligible. Tables 6.5 and 6.6 depict the spatial errors $e_{\rho}^{r}(t=2)$ and $e_{\mathbf{J}}^{r}(t=2)$ for the probability and current, respectively. Similarly, Table6.7 shows the temporal errors $e_{\Phi}^{r}(t=2)$ for the wave function under different $\tau$ and $\delta \in(0,1]$ with $h=h_{e}=\frac{1}{128}$ so that the spatial discretization error could be negligible. Tables 6.8 and 6.9 depict the temporal errors $e_{\rho}^{r}(t=2)$ and $e_{\mathbf{J}}^{r}(t=$ 2) for the probability and current, respectively.

From Tables 6.46.6 when $h \lesssim \delta$, spectral convergence (in space) is observed for the $S_{4 \mathrm{c}}$ method in the relative error for the wave function, probability and current. Similarly, from Tables 6.7.6.9. when $\tau \lesssim \delta$, fourth-order convergence (in time) is observed for the $S_{4 \mathrm{c}}$ method in the relative error for the wave function, probability and current. These suggest that the $\delta$-scalability for the $S_{4 \mathrm{c}}$ method in the semiclassical limit regime is: $h=O(\delta)$ and $\tau=O(\delta)$. In addition, noticing $\Phi=O(1), \rho=O(1)$ and $\mathbf{J}=O(1)$ when $0 \leq \delta \ll 1$, we can 


\begin{tabular}{cccccccc}
\hline & $h_{0}=1$ & $h_{0} / 2$ & $h_{0} / 2^{2}$ & $h_{0} / 2^{3}$ & $h_{0} / 2^{4}$ & $h_{0} / 2^{5}$ & $h_{0} / 2^{6}$ \\
\hline$\delta_{0}=1$ & $\mathbf{8 . 0 7 E}-1$ & $1.67 \mathrm{E}-1$ & $1.05 \mathrm{E}-2$ & $5.69 \mathrm{E}-6$ & $5.10 \mathrm{E}-10$ & $<1 \mathrm{E}-10$ & $<1 \mathrm{E}-10$ \\
\hline$\delta_{0} / 2$ & 1.45 & $\mathbf{6 . 8 9 E}-1$ & $4.28 \mathrm{E}-2$ & $6.46 \mathrm{E}-5$ & $3.06 \mathrm{E}-10$ & $<1 \mathrm{E}-10$ & $<1 \mathrm{E}-10$ \\
\hline$\delta_{0} / 2^{2}$ & 1.94 & 1.05 & $\mathbf{3 . 5 2 E}-1$ & $2.13 \mathrm{E}-3$ & $7.96 \mathrm{E}-10$ & $<1 \mathrm{E}-10$ & $<1 \mathrm{E}-10$ \\
\hline$\delta_{0} / 2^{3}$ & 2.52 & 1.03 & $7.07 \mathrm{E}-1$ & $\mathbf{1 . 2 4 E}-1$ & $7.75 \mathrm{E}-6$ & $8.16 \mathrm{E}-10$ & $<1 \mathrm{E}-10$ \\
\hline$\delta_{0} / 2^{4}$ & 2.85 & 1.77 & 1.10 & $5.84 \mathrm{E}-1$ & $\mathbf{4 . 7 2 E}-2$ & $6.75 \mathrm{E}-10$ & $<1 \mathrm{E}-10$ \\
\hline$\delta_{0} / 2^{5}$ & 3.88 & 4.06 & 1.11 & 1.07 & $3.81 \mathrm{E}-1$ & $\mathbf{1 . 2 2 E}-2$ & $5.63 \mathrm{E}-10$ \\
\hline
\end{tabular}

Table 6.6 Spatial errors $e_{\mathbf{J}}^{r}(t=2)$ of $S_{4 \mathrm{c}}$ under different $h$ and $\delta$ for the Dirac equation 1.17 in 1D in the semiclassical limit regime.

\begin{tabular}{cccccccc}
\hline & $\tau_{0}=1$ & $\tau_{0} / 2$ & $\tau_{0} / 2^{2}$ & $\tau_{0} / 2^{3}$ & $\tau_{0} / 2^{4}$ & $\tau_{0} / 2^{5}$ & $\tau_{0} / 2^{6}$ \\
\hline$\delta_{0}=1$ & $1.60 \mathrm{E}-1$ & $\mathbf{1 . 5 8 E - 2}$ & $5.09 \mathrm{E}-4$ & $2.08 \mathrm{E}-5$ & $1.27 \mathrm{E}-6$ & $7.89 \mathrm{E}-8$ & $4.94 \mathrm{E}-9$ \\
order & - & $\mathbf{3 . 3 4}$ & 4.96 & 4.61 & 4.04 & 4.01 & 4.00 \\
\hline$\delta_{0} / 2$ & $8.66 \mathrm{E}-1$ & $1.48 \mathrm{E}-1$ & $\mathbf{7 . 1 7 E}-3$ & $3.90 \mathrm{E}-4$ & $2.41 \mathrm{E}-5$ & $1.50 \mathrm{E}-6$ & $9.39 \mathrm{E}-8$ \\
order & - & 2.55 & $\mathbf{4 . 3 6}$ & 4.20 & 4.02 & 4.00 & 4.00 \\
\hline$\delta_{0} / 2^{2}$ & 1.26 & $9.52 \mathrm{E}-1$ & $1.38 \mathrm{E}-1$ & $\mathbf{7 . 3 8 E}-3$ & $4.50 \mathrm{E}-4$ & $2.80 \mathrm{E}-5$ & $1.75 \mathrm{E}-6$ \\
order & - & 0.40 & 2.78 & $\mathbf{4 . 2 3}$ & 4.03 & 4.01 & 4.00 \\
\hline$\delta_{0} / 2^{3}$ & 1.45 & 1.20 & $9.94 \mathrm{E}-1$ & $1.62 \mathrm{E}-1$ & $\mathbf{9 . 1 1 E - 3}$ & $5.57 \mathrm{E}-4$ & $3.46 \mathrm{E}-5$ \\
order & - & 0.27 & 0.27 & 2.62 & $\mathbf{4 . 1 5}$ & 4.03 & 4.01 \\
\hline$\delta_{0} / 2^{4}$ & 1.40 & 1.44 & 1.12 & $9.46 \mathrm{E}-1$ & $2.62 \mathrm{E}-1$ & $\mathbf{1 . 5 0 E - 2}$ & $9.15 \mathrm{E}-4$ \\
order & - & -0.04 & 0.36 & 0.25 & 1.85 & $\mathbf{4 . 1 3}$ & 4.03 \\
\hline$\delta_{0} / 2^{5}$ & 1.44 & 1.44 & 1.42 & 1.22 & 1.07 & $4.43 \mathrm{E}-1$ & $\mathbf{2 . 8 3 E - 2}$ \\
order & - & -0.01 & 0.03 & 0.22 & 0.19 & 1.27 & $\mathbf{3 . 9 7}$ \\
\hline
\end{tabular}

Table 6.7 Temporal errors $e_{\Phi}^{r}(t=2)$ of $S_{4 \mathrm{c}}$ under different $\tau$ and $\delta$ for the Dirac equation (1.17) in 1D in the semiclassical limit regime.

formally observe the following error bounds for $0<\delta \leq 1, \tau \lesssim \delta, h \lesssim \delta$ and $0 \leq n \leq \frac{T}{\tau}$

$$
\begin{aligned}
& \left\|\Phi^{n}-\Phi\left(t_{n}, \cdot\right)\right\|_{l^{2}} \lesssim \frac{h^{m_{0}}}{\delta^{m_{0}}}+\frac{\tau^{4}}{\delta^{4}}, \quad\left\|\rho^{n}-\rho\left(t_{n}, \cdot\right)\right\|_{l^{2}} \lesssim \frac{h^{m_{0}}}{\delta^{m_{0}}}+\frac{\tau^{4}}{\delta^{4}} \\
& \left\|\mathbf{J}^{n}-\mathbf{J}\left(t_{n}, \cdot\right)\right\|_{l^{2}} \lesssim \frac{h^{m_{0}}}{\delta^{m_{0}}}+\frac{\tau^{4}}{\delta^{4}} .
\end{aligned}
$$

where $m_{0} \geq 2$ depends on the regularity of the solution. Rigorous mathematical justification is still on-going.

6.3 In the simultaneously nonrelativistic and massless limit regime

We take $d=1, \delta=1$ and $v=\varepsilon$ in 1.17) with $\varepsilon \in(0,1]$. The initial data in 1.18) is taken as (5.2). In this parameter regime, the solution propagates waves with wavelength at $O(1)$ and $O(\varepsilon)$ in space and time, respectively. The problem is solved numerically on 


\begin{tabular}{cccccccc}
\hline & $\tau_{0}=1$ & $\tau_{0} / 2$ & $\tau_{0} / 2^{2}$ & $\tau_{0} / 2^{3}$ & $\tau_{0} / 2^{4}$ & $\tau_{0} / 2^{5}$ & $\tau_{0} / 2^{6}$ \\
\hline$\delta_{0}=1$ & $1.15 \mathrm{E}-1$ & $\mathbf{1 . 2 3 E - 2}$ & $4.11 \mathrm{E}-4$ & $1.70 \mathrm{E}-5$ & $1.03 \mathrm{E}-6$ & $6.40 \mathrm{E}-8$ & $4.11 \mathrm{E}-9$ \\
order & - & $\mathbf{3 . 2 3}$ & 4.90 & 4.59 & 4.05 & 4.01 & 3.96 \\
\hline$\delta_{0} / 2$ & $5.05 \mathrm{E}-1$ & $9.20 \mathrm{E}-2$ & $\mathbf{4 . 9 3 E - 3}$ & $2.36 \mathrm{E}-4$ & $1.44 \mathrm{E}-5$ & $8.98 \mathrm{E}-7$ & $5.62 \mathrm{E}-8$ \\
order & - & 2.45 & $\mathbf{4 . 2 2}$ & 4.39 & 4.03 & 4.01 & 4.00 \\
\hline$\delta_{0} / 2^{2}$ & $7.69 \mathrm{E}-1$ & $4.22 \mathrm{E}-1$ & $4.32 \mathrm{E}-2$ & $\mathbf{2 . 8 5 E - 3}$ & $1.73 \mathrm{E}-4$ & $1.08 \mathrm{E}-5$ & $6.72 \mathrm{E}-7$ \\
order & - & 0.86 & 3.29 & $\mathbf{3 . 9 2}$ & 4.04 & 4.01 & 4.00 \\
\hline$\delta_{0} / 2^{3}$ & 1.28 & $9.03 \mathrm{E}-1$ & $5.67 \mathrm{E}-1$ & $3.77 \mathrm{E}-2$ & $\mathbf{2 . 0 3 E - 3}$ & $1.23 \mathrm{E}-4$ & $7.66 \mathrm{E}-6$ \\
order & - & 0.51 & 0.67 & 3.91 & $\mathbf{4 . 2 1}$ & 4.04 & 4.01 \\
\hline$\delta_{0} / 2^{4}$ & $8.80 \mathrm{E}-1$ & 1.25 & $9.86 \mathrm{E}-1$ & $7.53 \mathrm{E}-1$ & $2.58 \mathrm{E}-2$ & $\mathbf{1 . 3 5 E - 3}$ & $8.15 \mathrm{E}-5$ \\
order & - & -0.50 & 0.34 & 0.39 & 4.87 & $\mathbf{4 . 2 6}$ & 4.05 \\
\hline$\delta_{0} / 2^{5}$ & $9.60 \mathrm{E}-1$ & $9.90 \mathrm{E}-1$ & 1.09 & 1.08 & $8.82 \mathrm{E}-1$ & $2.59 \mathrm{E}-2$ & $\mathbf{1 . 1 6 E - 3}$ \\
order & - & -0.04 & -0.14 & 0.02 & 0.29 & 5.09 & $\mathbf{4 . 4 8}$ \\
\hline
\end{tabular}

Table 6.8 Temporal errors $e_{\rho}^{r}(t=2)$ of $S_{4 \mathrm{c}}$ under different $\tau$ and $\delta$ for the Dirac equation (1.17) in 1D in the semiclassical limit regime.

\begin{tabular}{cccccccc}
\hline & $\tau_{0}=1$ & $\tau_{0} / 2$ & $\tau_{0} / 2^{2}$ & $\tau_{0} / 2^{3}$ & $\tau_{0} / 2^{4}$ & $\tau_{0} / 2^{5}$ & $\tau_{0} / 2^{6}$ \\
\hline$\delta_{0}=1$ & $1.98 \mathrm{E}-1$ & $\mathbf{2 . 2 1 E - 2}$ & $6.42 \mathrm{E}-4$ & $2.34 \mathrm{E}-5$ & $1.42 \mathrm{E}-6$ & $8.84 \mathrm{E}-8$ & $5.55 \mathrm{E}-9$ \\
order & - & $\mathbf{3 . 1 6}$ & 5.11 & 4.78 & 4.04 & 4.01 & 3.99 \\
\hline$\delta_{0} / 2$ & $6.61 \mathrm{E}-1$ & $1.93 \mathrm{E}-1$ & $\mathbf{8 . 7 2 E}-3$ & $4.34 \mathrm{E}-4$ & $2.67 \mathrm{E}-5$ & $1.66 \mathrm{E}-6$ & $1.04 \mathrm{E}-7$ \\
order & - & 1.78 & $\mathbf{4 . 4 7}$ & 4.33 & 4.02 & 4.01 & 4.00 \\
\hline$\delta_{0} / 2^{2}$ & 1.25 & $6.66 \mathrm{E}-1$ & $1.46 \mathrm{E}-1$ & $\mathbf{8 . 4 4 E - 3}$ & $5.16 \mathrm{E}-4$ & $3.21 \mathrm{E}-5$ & $2.00 \mathrm{E}-6$ \\
order & - & 0.91 & 2.19 & $\mathbf{4 . 1 2}$ & 4.03 & 4.01 & 4.00 \\
\hline$\delta_{0} / 2^{3}$ & 1.57 & 1.19 & $7.29 \mathrm{E}-1$ & $1.23 \mathrm{E}-1$ & $\mathbf{7 . 1 0 E}-3$ & $4.35 \mathrm{E}-4$ & $2.71 \mathrm{E}-5$ \\
order & - & 0.39 & 0.71 & 2.57 & $\mathbf{4 . 1 1}$ & 4.03 & 4.01 \\
\hline$\delta_{0} / 2^{4}$ & 1.04 & 1.47 & 1.15 & $8.24 \mathrm{E}-1$ & $9.50 \mathrm{E}-2$ & $\mathbf{5 . 8 6 E - 3}$ & $3.60 \mathrm{E}-4$ \\
order & - & -0.50 & 0.35 & 0.48 & 3.12 & $\mathbf{4 . 0 2}$ & 4.02 \\
\hline$\delta_{0} / 2^{5}$ & 1.02 & 1.14 & 1.19 & 1.19 & $9.39 \mathrm{E}-1$ & $7.34 \mathrm{E}-2$ & $\mathbf{5 . 2 2 E}-3$ \\
order & - & -0.16 & -0.06 & 0.01 & 0.34 & 3.68 & $\mathbf{3 . 8 1}$ \\
\hline
\end{tabular}

Table 6.9 Temporal errors $e_{\mathrm{J}}^{r}(t=2)$ of $S_{4 \mathrm{c}}$ under different $\tau$ and $\delta$ for the Dirac equation (1.17) in 1D in the semiclassical limit regime.

a bounded domain $\Omega=(-128,128)$, i.e. $a=-128$ and $b=128$ by $S_{4 \mathrm{c}}$. Similar to the nonrelativistic limit regime, the $S_{4 \mathrm{c}}$ method converges uniformly with respect to $\varepsilon \in(0,1]$ at spectral order in space. Detailed numerical results are omitted here for brevity. Here we only present temporal errors by taking $h=h_{e}=\frac{1}{16}$ so that the spatial discretization error could be negligible. Table 6.10 shows the temporal errors $e_{\Phi}^{r}(t=2)$ for the wave function under different $\tau$ and $\varepsilon \in(0,1]$. Similarly, Tables 6.11 and 6.12 depict the temporal errors $e_{\rho}^{r}(t=2)$ and $e_{\mathbf{J}}^{r}(t=2)$ for the probability and current, respectively. 


\begin{tabular}{cccccccc}
\hline & $\tau_{0}=1$ & $\tau_{0} / 2$ & $\tau_{0} / 2^{2}$ & $\tau_{0} / 2^{3}$ & $\tau_{0} / 2^{4}$ & $\tau_{0} / 2^{5}$ & $\tau_{0} / 2^{6}$ \\
\hline$\varepsilon_{0}=1$ & $\mathbf{1 . 1 2 E}-1$ & $4.20 \mathrm{E}-3$ & $2.18 \mathrm{E}-4$ & $1.33 \mathrm{E}-5$ & $8.30 \mathrm{E}-7$ & $5.18 \mathrm{E}-8$ & $3.24 \mathrm{E}-9$ \\
order & - & 4.74 & 4.27 & 4.03 & 4.01 & 4.00 & 4.00 \\
\hline$\varepsilon_{0} / 2$ & $4.72 \mathrm{E}-1$ & $\mathbf{3 . 6 6 E - 2}$ & $1.17 \mathrm{E}-3$ & $6.64 \mathrm{E}-5$ & $4.09 \mathrm{E}-6$ & $2.55 \mathrm{E}-7$ & $1.59 \mathrm{E}-8$ \\
order & - & $\mathbf{3 . 6 9}$ & 4.97 & 4.14 & 4.02 & 4.01 & 4.00 \\
\hline$\varepsilon_{0} / 2^{2}$ & 1.14 & $2.72 \mathrm{E}-1$ & $\mathbf{1 . 2 7 E}-2$ & $3.64 \mathrm{E}-4$ & $2.10 \mathrm{E}-5$ & $1.30 \mathrm{E}-6$ & $8.08 \mathrm{E}-8$ \\
order & - & 2.07 & $\mathbf{4 . 4 2}$ & 5.12 & 4.11 & 4.02 & 4.00 \\
\hline$\varepsilon_{0} / 2^{3}$ & 1.29 & $5.84 \mathrm{E}-1$ & $1.60 \mathrm{E}-1$ & $\mathbf{5 . 1 9 E}-3$ & $1.41 \mathrm{E}-4$ & $8.22 \mathrm{E}-6$ & $5.07 \mathrm{E}-7$ \\
order & - & 1.14 & 1.87 & $\mathbf{4 . 9 4}$ & 5.20 & 4.10 & 4.02 \\
\hline$\varepsilon_{0} / 2^{4}$ & 1.40 & $7.31 \mathrm{E}-1$ & $3.40 \mathrm{E}-1$ & $9.81 \mathrm{E}-2$ & $\mathbf{2 . 4 6 E}-3$ & $6.16 \mathrm{E}-5$ & $3.58 \mathrm{E}-6$ \\
order & - & 0.94 & 1.10 & 1.79 & $\mathbf{5 . 3 2}$ & 5.32 & 4.10 \\
\hline$\varepsilon_{0} / 2^{5}$ & 1.39 & 1.06 & $3.90 \mathrm{E}-1$ & $2.09 \mathrm{E}-1$ & $6.32 \mathrm{E}-2$ & $\mathbf{1 . 2 7 E}-3$ & $2.84 \mathrm{E}-5$ \\
order & - & 0.40 & 1.44 & 0.90 & 1.72 & $\mathbf{5 . 6 4}$ & 5.48 \\
\hline$\varepsilon_{0} / 2^{6}$ & 1.48 & 1.48 & $5.90 \mathrm{E}-1$ & $2.19 \mathrm{E}-1$ & $1.32 \mathrm{E}-1$ & $4.21 \mathrm{E}-2$ & $\mathbf{7 . 0 4 E}-4$ \\
order & - & 0.00 & 1.32 & 1.43 & 0.72 & 1.65 & $\mathbf{5 . 9 0}$ \\
\hline
\end{tabular}

Table 6.10 Temporal errors $e_{\Phi}^{r}(t=2)$ of $S_{4 \mathrm{c}}$ under different $\tau$ and $\varepsilon$ for the Dirac equation 1.17 in 1D in the simultaneously nonrelativistic and massless limit regime.

\begin{tabular}{cccccccc}
\hline & $\tau_{0}=1$ & $\tau_{0} / 2$ & $\tau_{0} / 2^{2}$ & $\tau_{0} / 2^{3}$ & $\tau_{0} / 2^{4}$ & $\tau_{0} / 2^{5}$ & $\tau_{0} / 2^{6}$ \\
\hline$\varepsilon_{0}=1$ & $\mathbf{8 . 6 2 E - 2}$ & $3.48 \mathrm{E}-3$ & $1.91 \mathrm{E}-4$ & $1.17 \mathrm{E}-5$ & $7.28 \mathrm{E}-7$ & $4.54 \mathrm{E}-8$ & $2.82 \mathrm{E}-9$ \\
order & - & 4.63 & 4.19 & 4.03 & 4.01 & 4.00 & 4.01 \\
\hline$\varepsilon_{0} / 2$ & $3.56 \mathrm{E}-1$ & $\mathbf{2 . 9 7 E - 2}$ & $7.90 \mathrm{E}-4$ & $4.56 \mathrm{E}-5$ & $2.82 \mathrm{E}-6$ & $1.76 \mathrm{E}-7$ & $1.10 \mathrm{E}-80$ \\
order & - & $\mathbf{3 . 5 9}$ & 5.23 & 4.12 & 4.01 & 4.00 & 4.00 \\
\hline$\varepsilon_{0} / 2^{2}$ & $9.98 \mathrm{E}-1$ & $2.83 \mathrm{E}-1$ & $\mathbf{1 . 2 2 E}-2$ & $2.54 \mathrm{E}-4$ & $1.45 \mathrm{E}-5$ & $8.95 \mathrm{E}-7$ & $5.57 \mathrm{E}-8$ \\
order & - & 1.82 & $\mathbf{4 . 5 3}$ & 5.59 & 4.13 & 4.02 & 4.01 \\
\hline$\varepsilon_{0} / 2^{3}$ & $8.15 \mathrm{E}-1$ & $5.58 \mathrm{E}-1$ & $1.60 \mathrm{E}-1$ & $\mathbf{4 . 1 8 E}-3$ & $9.00 \mathrm{E}-5$ & $5.29 \mathrm{E}-6$ & $3.27 \mathrm{E}-7$ \\
order & - & 0.55 & 1.80 & $\mathbf{5 . 2 6}$ & 5.54 & 4.09 & 4.02 \\
\hline$\varepsilon_{0} / 2^{4}$ & $9.32 \mathrm{E}-1$ & $7.05 \mathrm{E}-1$ & $3.32 \mathrm{E}-1$ & $1.02 \mathrm{E}-1$ & $\mathbf{1 . 6 9 E}-3$ & $3.69 \mathrm{E}-5$ & $2.19 \mathrm{E}-6$ \\
order & - & 0.40 & 1.09 & 1.70 & $\mathbf{5 . 9 2}$ & 5.52 & 4.08 \\
\hline$\varepsilon_{0} / 2^{5}$ & 1.05 & $6.88 \mathrm{E}-1$ & $3.28 \mathrm{E}-1$ & $2.07 \mathrm{E}-1$ & $6.70 \mathrm{E}-2$ & $\mathbf{8 . 6 8 E}-\mathbf{4}$ & $1.63 \mathrm{E}-5$ \\
order & - & 0.61 & 1.07 & 0.67 & 1.63 & $\mathbf{6 . 2 7}$ & 5.73 \\
\hline$\varepsilon_{0} / 2^{6}$ & $8.39 \mathrm{E}-1$ & $8.04 \mathrm{E}-1$ & $4.76 \mathrm{E}-1$ & $1.72 \mathrm{E}-1$ & $1.27 \mathrm{E}-1$ & $4.33 \mathrm{E}-2$ & $\mathbf{5 . 4 9 E}-4$ \\
order & - & 0.06 & 0.76 & 1.47 & 0.44 & 1.55 & $\mathbf{6 . 3 0}$ \\
\hline
\end{tabular}

Table 6.11 Temporal errors $e_{\rho}^{r}(t=2)$ of $S_{4 \mathrm{c}}$ under different $\tau$ and $\varepsilon$ for the Dirac equation 1.17 in 1D in the simultaneously nonrelativistic and massless limit regime.

From Tables 6.10 6.12 when $\tau \lesssim \varepsilon$, fourth-order convergence is observed for the $S_{4 \mathrm{c}}$ method in the relative error for the wave function, probability and current. This suggests that the $\varepsilon$-scalability for the $S_{4 \mathrm{c}}$ method in the simultaneously nonrelativistic and massless limit regime is: $h=O(1)$ and $\tau=O(\varepsilon)$. In addition, noticing $\Phi=O(1), \rho=O(1)$ and $\mathbf{J}=O\left(\varepsilon^{-1}\right)$ 


\begin{tabular}{cccccccc}
\hline & $\tau_{0}=1$ & $\tau_{0} / 2$ & $\tau_{0} / 2^{2}$ & $\tau_{0} / 2^{3}$ & $\tau_{0} / 2^{4}$ & $\tau_{0} / 2^{5}$ & $\tau_{0} / 2^{6}$ \\
\hline$\varepsilon_{0}=1$ & $\mathbf{2 . 0 3 E - 1}$ & $7.11 \mathrm{E}-3$ & $4.03 \mathrm{E}-4$ & $2.47 \mathrm{E}-5$ & $1.54 \mathrm{E}-6$ & $9.61 \mathrm{E}-8$ & $5.98 \mathrm{E}-9$ \\
order & - & 4.84 & 4.14 & 4.03 & 4.01 & 4.00 & 4.01 \\
\hline$\varepsilon_{0} / 2$ & $7.37 \mathrm{E}-1$ & $\mathbf{5 . 5 8 E - 2}$ & $1.89 \mathrm{E}-3$ & $1.11 \mathrm{E}-4$ & $6.84 \mathrm{E}-6$ & $4.26 \mathrm{E}-7$ & $2.66 \mathrm{E}-8$ \\
order & - & $\mathbf{3 . 7 2}$ & 4.88 & 4.09 & 4.02 & 4.00 & 4.00 \\
\hline$\varepsilon_{0} / 2^{2}$ & 1.34 & $4.30 \mathrm{E}-1$ & $\mathbf{1 . 8 1 E - 2}$ & $5.59 \mathrm{E}-4$ & $3.31 \mathrm{E}-5$ & $2.05 \mathrm{E}-6$ & $1.28 \mathrm{E}-7$ \\
order & - & 1.64 & $\mathbf{4 . 5 7}$ & 5.01 & 4.08 & 4.02 & 4.00 \\
\hline$\varepsilon_{0} / 2^{3}$ & 1.20 & $7.03 \mathrm{E}-1$ & $2.30 \mathrm{E}-1$ & $\mathbf{6 . 1 4 E}-3$ & $1.89 \mathrm{E}-4$ & $1.13 \mathrm{E}-5$ & $7.00 \mathrm{E}-7$ \\
order & - & 0.77 & 1.61 & $\mathbf{5 . 2 3}$ & 5.02 & 4.06 & 4.01 \\
\hline$\varepsilon_{0} / 2^{4}$ & 1.36 & 1.04 & $4.15 \mathrm{E}-1$ & $1.31 \mathrm{E}-1$ & $\mathbf{2 . 5 2 E - 3}$ & $7.59 \mathrm{E}-5$ & $4.57 \mathrm{E}-6$ \\
order & - & 0.39 & 1.32 & 1.66 & $\mathbf{5 . 7 1}$ & 5.05 & 4.05 \\
\hline$\varepsilon_{0} / 2^{5}$ & 1.63 & 1.32 & $5.79 \mathrm{E}-1$ & $2.47 \mathrm{E}-1$ & $8.28 \mathrm{E}-2$ & $\mathbf{1 . 2 7 E}-3$ & $3.26 \mathrm{E}-5$ \\
order & - & 0.30 & 1.19 & 1.23 & 1.58 & $\mathbf{6 . 0 3}$ & 5.28 \\
\hline$\varepsilon_{0} / 2^{6}$ & 1.38 & 1.47 & $8.97 \mathrm{E}-1$ & $3.04 \mathrm{E}-1$ & $1.52 \mathrm{E}-1$ & $5.54 \mathrm{E}-2$ & $\mathbf{7 . 5 2 E}-4$ \\
order & - & -0.09 & 0.71 & 1.56 & 1.00 & 1.45 & $\mathbf{6 . 2 0}$ \\
\hline
\end{tabular}

Table 6.12 Temporal errors $e_{\mathbf{J}}^{r}(t=2)$ of $S_{4 \mathrm{c}}$ under different $\tau$ and $\varepsilon$ for the Dirac equation 1.17 in 1D in the simultaneously nonrelativistic and massless limit regime.

when $0 \leq \varepsilon \ll 1$, we can formally observe the following error bounds for $0<\varepsilon \leq 1, \tau \lesssim \varepsilon$ and $0 \leq n \leq \frac{T}{\tau}$

$$
\begin{aligned}
& \left\|\Phi^{n}-\Phi\left(t_{n}, \cdot\right)\right\|_{l^{2}} \lesssim h^{m_{0}}+\frac{\tau^{4}}{\varepsilon^{3}}, \quad\left\|\rho^{n}-\rho\left(t_{n}, \cdot\right)\right\|_{l^{2}} \lesssim h^{m_{0}}+\frac{\tau^{4}}{\varepsilon^{3}}, \\
& \left\|\mathbf{J}^{n}-\mathbf{J}\left(t_{n}, \cdot\right)\right\|_{l^{2}} \lesssim \frac{1}{\varepsilon}\left(h^{m_{0}}+\frac{\tau^{4}}{\varepsilon^{3}}\right) .
\end{aligned}
$$

where $m_{0} \geq 2$ depends on the regularity of the solution. Rigorous mathematical justification is still on-going.

Based on the discussion in Section 1 and numerical comparison results in this section, Table 6.13 lists spatial/temporal wavelengths of the Dirac equation under different parameter regimes and the corresponding spatial/temporal resolution of the $S_{4 \mathrm{c}}$ method.

\section{Conclusion}

A new fourth-order compact time-splitting Fourier pseudospectral $\left(S_{4 \mathrm{c}}\right)$ method was proposed for the Dirac equation. It is explicit, fourth-order in time and spectral accurate in space. One major advantage in the method is to avoid using negative time steps in integrating sub-problems via the double commutator. Numerical results showed that it is much more accurate than first-order and second-order time-splitting methods, and it is more accurate 


\begin{tabular}{c|c|c|c|c|c|c}
\hline & $\begin{array}{c}\text { Spatial } \\
\text { wavelength }\end{array}$ & $\begin{array}{c}\text { Temporal } \\
\text { wavelength }\end{array}$ & $\begin{array}{c}\text { Spatial } \\
\text { accuracy }\end{array}$ & $\begin{array}{c}\text { Temporal } \\
\text { accuracy }\end{array}$ & $\begin{array}{c}\text { Spatial } \\
\text { resolution }\end{array}$ & $\begin{array}{c}\text { Temporal } \\
\text { resolution }\end{array}$ \\
\hline $\begin{array}{c}\text { Standard } \\
\text { regime }\end{array}$ & $O(1)$ & $O(1)$ & spectral & $O\left(\tau^{4}\right)$ & $O(1)$ & $O(1)$ \\
\hline $\begin{array}{c}\text { Nonrelativistic } \\
\text { limit regime }\end{array}$ & $O(1)$ & $O\left(\varepsilon^{2}\right)$ & spectral & $O\left(\frac{\tau^{4}}{\varepsilon^{6}}\right)$ & $O(1)$ & $O\left(\varepsilon^{2}\right)$ \\
\hline $\begin{array}{c}\text { Semiclassical } \\
\text { limit regime }\end{array}$ & $O(\delta)$ & $O(\delta)$ & spectral & $O\left(\frac{\tau^{4}}{\delta^{4}}\right)$ & $O(\delta)$ & $O(\delta)$ \\
\hline $\begin{array}{c}\text { Nonrelativistic } \\
\text { \&massless } \\
\text { limit regime }\end{array}$ & $O(1)$ & $O(\varepsilon)$ & spectral & $O\left(\frac{\tau^{4}}{\varepsilon^{3}}\right)$ & $O(1)$ & $O(\varepsilon)$ \\
\hline $\begin{array}{c}\text { Massless } \\
\text { limit regime }\end{array}$ & $O(1)$ & $O(1)$ & spectral & $O\left(\tau^{4}\right)$ & $O(1)$ & $O(1)$ \\
\hline
\end{tabular}

Table 6.13 Spatial/temporal wavelengths of the Dirac equation under different parameter regimes and the corresponding spatial/temporal resolution of the $S_{4 \mathrm{c}}$ method.

than the standard fourth-order time-splitting method and is more efficient than the partitioned Runge-Kutta time-splitting method, especially in 1D or in high dimensions without magnetic potentials. In addition, it is very robust for simulating long time dynamics. Spatial and temporal resolution of the proposed numerical method was studied numerically for the Dirac equation under different parameter regimes including the nonrelativistic limit regime, the semiclassical limit regime, and the simultaneously nonrelativistic and massless limit regime. Based on our extensive numerical results, for numerical simulation of the dynamics of the Dirac equation in 1D or in high dimensions without magnetic potential, the $S_{4 \mathrm{c}}$ method is a very efficient and accurate as well as simple numerical method. Of course, for the Dirac equation in high dimensions with magnetic potential, $S_{4 \mathrm{RK}}$ is a good choice.

\section{References}

1. D. A. Abanin, S. V. Morozov, L. A. Ponomarenko, R. V. Gorbachev, A. S. Mayorov, M. I. Katsnelson, K. Watanabe, T. Taniguchi, K. S. Novoselov, L. S. Levitov and A. K. Geim, Giant nonlocality near the Dirac point in graphene, Science, 332, 328-330 (2011)

2. X. Antoine, W. Bao and C. Besse, Computational methods for the dynamics of the nonlinear Schrodinger/Gross-Pitaevskii equations, Comput. Phys. Commun., 184, 2621-2633 (2013)

3. X. Antoine and E. Lorin, Computational performance of simple and efficient sequential and parallel Dirac equation solvers, Hal-01496817 (2017)

4. X. Antoine, E. Lorin, J. Sater, F. Fillion-Gourdeau, A. D. Bandrauk, Absorbing boundary conditions for relativistic quantum mechanics equations, J. Comput. Phys., 277, 268304 (2014)

5. A. Arnold and H. Steinrück, The 'electromagnetic' Wigner equation for an electron with spin, ZAMP, 40, 793-815 (1989)

6. P. Bader, A. Iserles, K. Kropielnicka and P. Singh, Effective approximation for the linear time-dependent Schrödinger equation, Found. Comp. Math., 14, 689-720 (2014)

7. W. Bao and Y. Cai, Mathematical theory and numerical methods for Bose-Einstein condensation, Kinet. Relat. Mod., 6, 1-135 (2013) 
8. W. Bao, Y. Cai, X. Jia and Q. Tang, A uniformly accurate multiscale time integrator pseudospectral method for the Dirac equation in the nonrelativistic limit regime, SIAM J. Numer. Anal., 54, 1785-1812 (2016)

9. W. Bao, Y. Cai, X. Jia and Q. Tang, Numerical methods and comparison for the Dirac equation in the nonrelativistic limit regime, J. Sci. Comput., 71, 1094-1134 (2017)

10. W. Bao, Y. Cai, X. Jia and J. Yin, Error estimates of numerical methods for the nonlinear Dirac equation in the nonrelativistic limit regime, Sci. China Math., 59, 1461-1494 (2016)

11. W. Bao, S. Jin and P. A. Markowich, On time-splitting spectral approximation for the Schrödinger equation in the semiclassical regime, J. Comput. Phys., 175, 487-524 (2002)

12. W. Bao, S. Jin and P. A. Markowich, Numerical study of time-splitting spectral discretizations of nonlinear Schrödinger equations in the semi-classical regimes, SIAM J. Sci. Comput., 25, 27-64 (2003)

13. W. Bao and X. Li, An efficient and stable numerical methods for the Maxwell-Dirac system, J. Compute. Phys., 199, 663-687 (2004)

14. W. Bao and J. Shen, A fourth-order time-splitting Laguerre-Hermite pseudo-spectral method for BoseEinstein condensates, SIAM J. Sci. Comput., 26, 2010-2028 (2005)

15. P. Bechouche, N. Mauser and F. Poupaud, (Semi)-nonrelativistic limits of the Dirac equaiton with external time-dependent electromagnetic field, Commun. Math. Phys., 197, 405-425 (1998)

16. S. Blanesa, P. C. Moan, Practical symplectic partitioned Runge-Kutta and Runge-Kutta-Nyström methods, J. Comput. Appl. Math., 142, 313-330 (2002)

17. O. Boada, A. Celi, J. I. Latorre and M. Lewenstein, Dirac equation for cold atoms in artificial curved spacetimes, New J. Phys., 13, 035002 (2011)

18. J. Bolte, and S. Keppeler, A semiclassical approach to the Dirac equation, Ann. Phys., 274, 125-162 (1999)

19. M. Caliari, A. Ostermann, C. Piazzola, A splitting approach for the magnetic Schrödinger equation, J. Comput. Appl. Math., 316, 74-85 (2017)

20. J.-J. Cao, X.-G. Li, J.-L. Qiu and J.-J. Zhang, Time-compact scheme for the one-dimensional Dirac equation, Discrete Dynamics in Nature and Society, 2016 (2016), article 3670139.

21. R. Carles, On Fourier time-splitting methods for nonlinear Schrödinger equations in the semi-classical limit, SIAM J. Numer. Anal., 51, 3232-3258 (2013)

22. R. Carles and C. Gallo, On Fourier time-splitting methods for nonlinear Schrödinger equations in the semi-classical limit II. Analytic regularity, Numer. Math., 136, 315-342 (2017)

23. S. A. Chin, Symplectic integrators from composite operator factorizations, Phys. Lett. A, 226, 344-348 (1997)

24. S. A. Chin, C. R. Chen, Fourth order gradient symplectic integrator methods for solving the timedependent Schrödinger equation, J. Chem. Phys., 114, 7338-7341 (2001)

25. S. A. Chin, C. R. Chen, Gradient symplectic algorithms for solving the Schrödinger equation with timedependent potentials, J. Chem. Phys., 117, 1409-1415 (2002)

26. A. Das, General solutions of MaxwellDirac equations in $1+1$ dimensional space-time and spatialconfined solution, J. Math. Phys., 34, 3986-3999 (1993)

27. A. Das, D. Kay, A class of exact plane wave solutions of the MaxwellDirac equations, J. Math. Phys., 30, 2280-2284 (1989)

28. A. S. Davydov, Quantum mechanics, Pergamon Press (1976)

29. P. A. M. Dirac, The quantum theory of the electron, Proc. R. Soc. Lond. A, 117, 610-624 (1928)

30. P. A. M. Dirac, A theory of electrons and protons, Proc. R. Soc. Lond. A, 126, 360-365 (1930)

31. P. A. M. Dirac, The principles of quantum mechanics, third ed., Oxford University Press, London (1947)

32. M. Esteban and E. Séré, Existence and multiplicity of solutions for linear and nonlinear Dirac problems, Partial Differential Equations and Their Applications (1997)

33. C. L. Fefferman and M. I. Weistein, Honeycomb lattice potentials and Dirac points, J. Am. Math. Soc., $25,11691220(2012)$ 
34. C. L. Fefferman and M. I. Weistein, Wave packets in honeycomb structures and two-dimensional Dirac equations, Commun. Math. Phys., 326, 251-286 (2014)

35. A. Ferreira, J. V. Gomes, J. Nilsson, E. R. Mucciolo, N. M. R. Peres, A. H. Catro Neto, Unifieddescription of the dc-conductivity of monolayer and bilayer graphene at finite densities based on resonant scatterers, Phys. Rev. B, 83, 165402 (2011)

36. F. Fillion-Gourdeau, E. Lorin, and A. D. Bandrauk, Resonantly Enhanced Pair Production in a Simple Diatomic Model, Phys. Rev. Lett., 110, 013002 (2013)

37. E. Forest, R. D. Ruth, Fourth-order symplectic integration, Physica D: Nonlinear Phenomena, 43, 105117 (1990)

38. S. Geng, Syplectic partitioned Runge-Kutta methods, J. Comput. Math., 11, 365-372 (1993)

39. P. Gérad, P. A. Markowich, N. J. Mauser, and F. Poupaud, Homogenization limits and Wigner transforms, Comm. Pure Appl. Math., 50, 321-377 (1997)

40. F. Gesztesy, H. Grosse, B. Thaller, A rigorious approach to relativistic corrections of bound state energies for spin-1/2 particles, Ann. Inst. Henri Poincaré Phys. Theor., 40, 159-174 (1984)

41. L. Gross, The Cauchy problem for the coupled Maxwell and Dirac equations, Commun. Pure Appl. Math., 19, 1-15 (1966)

42. Z. Huang, S. Jin, P. A. Markowich, C. Sparber and C. Zheng, A time-splitting spectral scheme for the Maxwell-Dirac system, J. Comput. Phys., 208, 761-789 (2005)

43. W. Hunziker, On the nonrelativistic limit of the Dirac theory, Commun. Math. Phys., 40, 215-222 (1975)

44. S. Jiang, L. Greengard and W. Bao, Fast and accurate evaluation of nonlocal Coulomb and dipole-dipole interactions via the nonuniform FFT, SIAM J. Sci. Comput., 36, B777-B794 (2014)

45. S. Jin, P. Markowich and C. Sparber, Mathematical and numerical methods for semiclassical Schrödinger equations, Acta Numer., 20, 121-209 (2011)

46. M. Lemou, F. Méhats and X. Zhao, Uniformly accurate numerical schemes for the nonlinear Dirac equation in the nonrelativistic limit regime, arXiv:1605.02475](2016)

47. R. I. McLachlan, G. R. W. Quispel, Splitting methods, Acta Numer., 341-434 (2002)

48. A. H. C. Neto, F. Guinea, N. M. R. Peres, K. S. Novoselov and A. K. Geim, The electronic properties of graphene, Rev. Mod. Phys., 81, 109-162 (2009)

49. K. S. Novoselov, A. K. Geim, S.V. Morozov, D. Jiang, Y. Zhang, S. V. Dubonos, I. V. Grigorieva, A. A. Firsov, Electric Field Effect in Atomically Thin Carbon Films, Science, 306, 666-669 (2004)

50. J. W. Nraun, Q. Su and R. Grobe, Numerical approach to solve the time-dependent Dirac equation, Phys. Rev. A, 59, 604-612 (1999)

51. T. Ohlsson, Relativistic Quantum Physics: From Advanced Quantum Mechanics to Introductory Quantum Field Theory, Cambridge University Press, the United Kingdom (2011)

52. P. Ring, Relativistic mean field theory in finite nuclei, Prog. Part. Nucl. Phys., 37, 193-263 (1996)

53. H. Spohn, Semiclassical limit of the Dirac equation and spin precession, Annal. Phys., 282, 420-431 (2000)

54. G. Strang, On the construction and comparison of difference schemes, SIAM J. Numer. Anal., 5, 507-517 (1968)

55. M. Suzuki, Fractal decomposition of exponential operators with applications to many-body theories and Monte Carlo simulations, Phys. Lett. A, 146, 319-323 (1990)

56. M. Suzuki, General theory of fractal path integrals with applications to manybody theories and statistical physics, J. Math. Phys., 32, 400-407 (1991)

57. M. Suzuki, General decompositon theory of ordered exponentials, Proc. Japan Acad., 69, 161-166 (1993)

58. M. Suzuki, New scheme of hybrid exponential product formulas with applications to quantum MonteCarlo Simulations, Springer Proc. Phys., 80, 169-174 (1995)

59. M. Thalhammer Convergence analysis of high-order time-splitting pseudo-spectral methods for nonlinear Schrödinger equations, SIAM J. Numer. Anal., 50, 3231-3258 (2012)

60. B. Thaller, The Dirac equation, Springer, New York (1992) 
61. H. F. Trotter, On the product of semi-groups of operators, Proc. Amer. Math. Soc., 10, 545-551 (1959)

62. H. Wu, Z. Huang, S. Jin and D. Yin, Gaussian beam methods for the Dirac equation in the semi-classical regime, Commun. Math. Sci., 10, 1301-1305 (2012)

63. S. Xu, I. Belopolski, N. Alidoust, M. Neupane, G. Bian, C. Zhang, R. Sankar, G. Chang, Z. Yuan, C. Lee, S. Huang, H. Zheng, J. Ma, D. S. Sanchez, B. Wang, A. Bansil, F. Chou, P. P. Shibayev, H. Lin, S. Jia, M. Z. Hasan, Discovery of a Weyl fermion semimetal and topological Fermi arcs, Science, 349, 613-617 (2015)

64. H. Yoshida, Construction of higher order symplectic integrators, Phys. Lett. A, 150, 262-268 (1990)

Appendix A. Proof of Lemma 3.3 on double commutator of the Dirac equation in 2D

Proof Combining (3.12) and (3.2), we obtain

$$
[W,[T, W]]=-\frac{1}{\varepsilon}\left[W,\left[\sigma_{1} \partial_{1}, W\right]\right]-\frac{1}{\varepsilon}\left[W,\left[\sigma_{2} \partial_{2}, W\right]\right]-\frac{i v}{\delta \varepsilon^{2}}\left[W,\left[\sigma_{3}, W\right]\right] .
$$

From (1.3), we have

$$
\begin{aligned}
& \sigma_{j}^{2}=I_{2}, \quad \sigma_{j} \sigma_{l}=-\sigma_{l} \sigma_{j}, \quad 1 \leq j \neq l \leq 3 \\
& \sigma_{1} \sigma_{2}=i \sigma_{3}, \quad \sigma_{2} \sigma_{3}=i \sigma_{1}, \quad \sigma_{3} \sigma_{1}=i \sigma_{2}
\end{aligned}
$$

Noticing 3.12, 3.1 and (A.2), we get

$$
\begin{aligned}
& {\left[W,\left[\sigma_{1} \partial_{1}, W\right]\right] } \\
&=-\frac{1}{\delta^{2}}\left(2\left(V(\mathbf{x}) I_{2}-A_{1}(\mathbf{x}) \sigma_{1}-A_{2}(\mathbf{x}) \sigma_{2}\right)\left(\sigma_{1} \partial_{1}\right)\left(V(\mathbf{x}) I_{2}-A_{1}(\mathbf{x}) \sigma_{1}-A_{2}(\mathbf{x}) \sigma_{2}\right)\right. \\
&\left.-\left(V(\mathbf{x}) I_{2}-A_{1}(\mathbf{x}) \sigma_{1}-A_{2}(\mathbf{x}) \sigma_{2}\right)^{2}\left(\sigma_{1} \partial_{1}\right)-\left(\sigma_{1} \partial_{1}\right)\left(V(\mathbf{x}) I_{2}-A_{1}(\mathbf{x}) \sigma_{1}-A_{2}(\mathbf{x}) \sigma_{2}\right)^{2}\right) \\
&=-\frac{2}{\delta^{2}} \sigma_{1} A_{2}(\mathbf{x}) \sigma_{2}\left(\partial_{1} V(\mathbf{x}) I_{2}-\partial_{1} A_{1}(\mathbf{x}) \sigma_{1}-\partial_{1} A_{2}(\mathbf{x}) \sigma_{2}\right) \\
&-\frac{2}{\delta^{2}} \sigma_{1}\left(V(\mathbf{x}) I_{2}-A_{1}(\mathbf{x}) \sigma_{1}+A_{2}(\mathbf{x}) \sigma_{2}\right)\left(V(\mathbf{x}) I_{2}-A_{1}(\mathbf{x}) \sigma_{1}-A_{2}(\mathbf{x}) \sigma_{2}\right) \partial_{1} \\
&+\frac{1}{\delta^{2}} \sigma_{1}\left(V(\mathbf{x}) I_{2}-A_{1}(\mathbf{x}) \sigma_{1}+A_{2}(\mathbf{x}) \sigma_{2}\right)^{2} \partial_{1}+\frac{1}{\delta^{2}} \sigma_{1}\left(V(\mathbf{x}) I_{2}-A_{1}(\mathbf{x}) \sigma_{1}-A_{2}(\mathbf{x}) \sigma_{2}\right)^{2} \partial_{1} \\
&-\frac{2}{\delta^{2}} \sigma_{1} A_{2}(\mathbf{x}) \sigma_{2}\left(\partial_{1} V(\mathbf{x}) I_{2}-\partial_{1} A_{1}(\mathbf{x}) \sigma_{1}-\partial_{1} A_{2}(\mathbf{x}) \sigma_{2}\right) \\
&=-\frac{4}{\delta^{2}} A_{2}(\mathbf{x})\left(\partial_{1} V(\mathbf{x}) \sigma_{1} \sigma_{2}+\partial_{1} A_{1}(\mathbf{x}) \sigma_{2}-\partial_{1} A_{2}(\mathbf{x}) \sigma_{1}\right)+\frac{4}{\delta^{2}} A_{2}^{2}(\mathbf{x}) \sigma_{1} \partial_{1} \\
&-\frac{4}{\delta^{2}} A_{1}(\mathbf{x}) A_{2}(\mathbf{x}) \sigma_{2} \partial_{1} \\
&= \frac{4}{\delta^{2}}\left(A_{2}^{2}(\mathbf{x}) \sigma_{1}-A_{1}(\mathbf{x}) A_{2}(\mathbf{x}) \sigma_{2}\right) \partial_{1}+\frac{4}{\delta^{2}} A_{2}(\mathbf{x})\left(\partial_{1} A_{2}(\mathbf{x}) \sigma_{1}-\partial_{1} A_{1}(\mathbf{x}) \sigma_{2}\right) \\
&-\frac{4 i}{\delta^{2}} A_{2}(\mathbf{x}) \partial_{1} V(\mathbf{x}) \sigma_{3} .
\end{aligned}
$$




$$
\begin{aligned}
{\left[W,\left[\sigma_{3}, W\right]\right]=} & -\frac{1}{\delta^{2}}\left(2\left(V(\mathbf{x}) I_{2}-A_{1}(\mathbf{x}) \sigma_{1}-A_{2}(\mathbf{x}) \sigma_{2}\right) \sigma_{3}\left(V(\mathbf{x}) I_{2}-A_{1}(\mathbf{x}) \sigma_{1}-A_{2}(\mathbf{x}) \sigma_{2}\right)\right. \\
& \left.-\left(V(\mathbf{x}) I_{2}-A_{1}(\mathbf{x}) \sigma_{1}-A_{2}(\mathbf{x}) \sigma_{2}\right)^{2} \sigma_{3}-\sigma_{3}\left(V(\mathbf{x}) I_{2}-A_{1}(\mathbf{x}) \sigma_{1}-A_{2}(\mathbf{x}) \sigma_{2}\right)^{2}\right) \\
= & \frac{2}{\delta^{2}} \sigma_{3}\left(V(\mathbf{x}) I_{2}+A_{1}(\mathbf{x}) \sigma_{1}+A_{2}(\mathbf{x}) \sigma_{2}\right)\left(A_{1}(\mathbf{x}) \sigma_{1}+A_{2}(\mathbf{x}) \sigma_{2}\right) \\
& -\frac{2}{\delta^{2}} \sigma_{3}\left(A_{1}(\mathbf{x}) \sigma_{1}+A_{2}(\mathbf{x}) \sigma_{2}\right)\left(V(\mathbf{x}) I_{2}-A_{1}(\mathbf{x}) \sigma_{1}-A_{2}(\mathbf{x}) \sigma_{2}\right) \\
= & \frac{4}{\delta^{2}}\left(A_{1}^{2}(\mathbf{x})+A_{2}^{2}(\mathbf{x})\right) \sigma_{3} .
\end{aligned}
$$

$\left[W,\left[\sigma_{2} \partial_{2}, W\right]\right]=-\frac{4}{\delta^{2}}\left(A_{1}(\mathbf{x}) A_{2}(\mathbf{x}) \sigma_{1}-A_{1}^{2}(\mathbf{x}) \sigma_{2}\right) \partial_{2}-\frac{4}{\delta^{2}} A_{1}(\mathbf{x})\left(\partial_{2} A_{2}(\mathbf{x}) \sigma_{1}-\partial_{2} A_{1}(\mathbf{x}) \sigma_{2}\right)$

$$
+\frac{4 i}{\delta^{2}} A_{1}(\mathbf{x}) \partial_{2} V(\mathbf{x}) \sigma_{3} \text {. }
$$

Plugging (A.3), (A.5) and (A.4) into (A.1), after some computation, we can get (3.13).

Appendix B. Proof of Lemma 3.4 on double commutator of the Dirac equation in 3D

Proof Combining (3.17) and 3.2), we obtain

$$
[W,[T, W]]=-\frac{1}{\varepsilon}\left[W,\left[\alpha_{1} \partial_{1}, W\right]\right]-\frac{1}{\varepsilon}\left[W,\left[\alpha_{2} \partial_{2}, W\right]\right]-\frac{1}{\varepsilon}\left[W,\left[\alpha_{3} \partial_{3}, W\right]\right]-\frac{i v}{\delta \varepsilon^{2}}[W,[\beta, W]] .
$$

From (1.2) and 3.16, we have

$$
\begin{aligned}
& \beta^{2}=I_{4}, \quad \alpha_{j}^{2}=I_{4}, \quad \alpha_{j} \alpha_{l}=-\alpha_{l} \alpha_{j}, \\
& \beta \alpha_{j}=-\alpha_{j} \beta, \quad \gamma \alpha_{j}=\alpha_{j} \gamma, \quad 1 \leq j \neq l \leq 3, \\
& \alpha_{1} \alpha_{2}=i \gamma \alpha_{3}, \quad \alpha_{2} \alpha_{3}=i \gamma \alpha_{1}, \quad \alpha_{3} \alpha_{1}=i \gamma \alpha_{2} .
\end{aligned}
$$

Noticing 3.17, 3.1 and (B.2), we get

$$
\begin{aligned}
{[W,[\beta, W]]=} & -\frac{1}{\delta^{2}}\left(2\left(V(\mathbf{x}) I_{4}-\sum_{j=1}^{3} A_{j}(\mathbf{x}) \alpha_{j}\right) \beta\left(V(\mathbf{x}) I_{4}-\sum_{j=1}^{3} A_{j}(\mathbf{x}) \alpha_{j}\right)\right. \\
& \left.-\left(V(\mathbf{x}) I_{4}-\sum_{j=1}^{3} A_{j}(\mathbf{x}) \alpha_{j}\right)^{2} \beta-\beta\left(V(\mathbf{x}) I_{4}-\sum_{j=1}^{3} A_{j}(\mathbf{x}) \alpha_{j}\right)^{2}\right) \\
= & -\frac{2}{\delta^{2}} \beta\left(V(\mathbf{x}) I_{4}+\sum_{j=1}^{3} A_{j}(\mathbf{x}) \alpha_{j}\right)\left(V(\mathbf{x}) I_{4}-\sum_{j=1}^{3} A_{j}(\mathbf{x}) \alpha_{j}\right) \\
& +\frac{1}{\delta^{2}} \beta\left(V(\mathbf{x}) I_{4}+\sum_{j=1}^{3} A_{j}(\mathbf{x}) \alpha_{j}\right)^{2}+\frac{1}{\delta^{2}} \beta\left(V(\mathbf{x}) I_{4}-\sum_{j=1}^{3} A_{j}(\mathbf{x}) \alpha_{j}\right)^{2} \\
= & \frac{4}{\delta^{2}}\left(A_{1}^{2}(\mathbf{x})+A_{2}^{2}(\mathbf{x})+A_{3}^{2}(\mathbf{x})\right) \beta .
\end{aligned}
$$




$$
\begin{aligned}
{\left[W,\left[\alpha_{1} \partial_{1}, W\right]\right] } \\
=-\frac{1}{\delta^{2}}\left(2\left(V(\mathbf{x}) I_{4}-\sum_{j=1}^{3} A_{j}(\mathbf{x}) \alpha_{j}\right)\left(\alpha_{1} \partial_{1}\right)\left(V(\mathbf{x}) I_{4}-\sum_{j=1}^{3} A_{j}(\mathbf{x}) \alpha_{j}\right)\right. \\
\left.-\left(V(\mathbf{x}) I_{4}-\sum_{j=1}^{3} A_{j}(\mathbf{x}) \alpha_{j}\right)^{2}\left(\alpha_{1} \partial_{1}\right)-\left(\alpha_{1} \partial_{1}\right)\left(V(\mathbf{x}) I_{4}-\sum_{j=1}^{3} A_{j}(\mathbf{x}) \alpha_{j}\right)^{2}\right) \\
=-\frac{4}{\delta^{2}} \alpha_{1}\left(A_{2}(\mathbf{x}) \alpha_{2}+A_{3}(\mathbf{x}) \alpha_{3}\right)\left(\partial_{1} V(\mathbf{x}) I_{4}-\partial_{1} A_{1}(\mathbf{x}) \alpha_{1}-\partial_{1} A_{2}(\mathbf{x}) \alpha_{2}-\partial_{1} A_{3}(\mathbf{x}) \alpha_{3}\right) \\
+\frac{1}{\delta^{2}} \alpha_{1}\left(\left(V(\mathbf{x}) I_{4}-A_{1}(\mathbf{x}) \alpha_{1}+A_{2}(\mathbf{x}) \alpha_{2}+A_{3}(\mathbf{x}) \alpha_{3}\right)^{2}+\left(V(\mathbf{x}) I_{4}-\sum_{j=1}^{3} A_{j}(\mathbf{x}) \alpha_{j}\right)^{2}\right. \\
\left.-2\left(V(\mathbf{x}) I_{4}-A_{1}(\mathbf{x}) \alpha_{1}+A_{2}(\mathbf{x}) \alpha_{2}+A_{3}(\mathbf{x}) \alpha_{3}\right)\left(V(\mathbf{x}) I_{4}-\sum_{j=1}^{3} A_{j}(\mathbf{x}) \alpha_{j}\right)\right) \partial_{1}, \\
=\frac{4}{\delta^{2}}\left(A_{2}(\mathbf{x}) \alpha_{2}+A_{3}(\mathbf{x}) \alpha_{3}\right) \alpha_{1}\left(\partial_{1} V(\mathbf{x}) I_{4}-\partial_{1} A_{1}(\mathbf{x}) \alpha_{1}-\partial_{1} A_{2}(\mathbf{x}) \alpha_{2}-\partial_{1} A_{3}(\mathbf{x}) \alpha_{3}\right) \\
+\frac{4}{\delta^{2}}\left(\left(A_{2}^{2}(\mathbf{x})+A_{3}^{2}(\mathbf{x})\right) \alpha_{1}-A_{1}(\mathbf{x}) A_{2}(\mathbf{x}) \alpha_{2}-A_{1}(\mathbf{x}) A_{3}(\mathbf{x}) \alpha_{3}\right) \partial_{1} \\
=\frac{4}{\delta^{2}}\left(\left(A_{2}(\mathbf{x}) \partial_{1} A_{2}(\mathbf{x})+A_{3}(\mathbf{x}) \partial_{1} A_{3}(\mathbf{x})\right) \alpha_{1}-A_{2}(\mathbf{x}) \partial_{1} A_{1}(\mathbf{x}) \alpha_{2}-A_{3}(\mathbf{x}) \partial_{1} A_{1}(\mathbf{x}) \alpha_{3}\right. \\
\left.+\left(i A_{2}(\mathbf{x}) \partial_{1} A_{3}(\mathbf{x})-i A_{3}(\mathbf{x}) \partial_{1} A_{2}(\mathbf{x})\right) \gamma+i A_{3}(\mathbf{x}) \partial_{1} V(\mathbf{x}) \gamma \alpha_{2}-i A_{2}(\mathbf{x}) \partial_{1} V(\mathbf{x}) \gamma \alpha_{3}\right) \\
+\frac{4}{\delta^{2}}\left(\left(A_{2}^{2}(\mathbf{x})+A_{3}^{2}(\mathbf{x})\right) \alpha_{1}-A_{1}(\mathbf{x}) A_{2}(\mathbf{x}) \alpha_{2}-A_{1}(\mathbf{x}) A_{3}(\mathbf{x}) \alpha_{3}\right) \partial_{1} .
\end{aligned}
$$

$\left[W,\left[\alpha_{2} \partial_{2}, W\right]\right]$

$$
\begin{aligned}
= & \frac{4}{\delta^{2}}\left(-A_{1}(\mathbf{x}) \partial_{2} A_{2}(\mathbf{x}) \alpha_{1}+\left(A_{1}(\mathbf{x}) \partial_{2} A_{1}(\mathbf{x})+A_{3}(\mathbf{x}) \partial_{2} A_{3}(\mathbf{x})\right) \alpha_{2}-A_{3}(\mathbf{x}) \partial_{2} A_{2}(\mathbf{x}) \alpha_{3}\right. \\
& \left.+\left(i A_{3}(\mathbf{x}) \partial_{2} A_{1}(\mathbf{x})-i A_{1}(\mathbf{x}) \partial_{2} A_{3}(\mathbf{x})\right) \gamma-i A_{3}(\mathbf{x}) \partial_{2} V(\mathbf{x}) \gamma \alpha_{1}+i A_{1}(\mathbf{x}) \partial_{2} V(\mathbf{x}) \gamma \alpha_{3}\right) \\
& +\frac{4}{\delta^{2}}\left(\left(A_{1}^{2}(\mathbf{x})+A_{3}^{2}(\mathbf{x})\right) \alpha_{2}-A_{2}(\mathbf{x}) A_{1}(\mathbf{x}) \alpha_{1}-A_{2}(\mathbf{x}) A_{3}(\mathbf{x}) \alpha_{3}\right) \partial_{2} .
\end{aligned}
$$

$$
\begin{aligned}
& {\left[W,\left[\alpha_{3} \partial_{3}, W\right]\right]} \\
& =\frac{4}{\delta^{2}}\left(-A_{1}(\mathbf{x}) \partial_{3} A_{3}(\mathbf{x}) \alpha_{1}-A_{2}(\mathbf{x}) \partial_{3} A_{3}(\mathbf{x}) \alpha_{2}+\left(A_{1}(\mathbf{x}) \partial_{3} A_{1}(\mathbf{x})+A_{2}(\mathbf{x}) \partial_{3} A_{2}(\mathbf{x})\right) \alpha_{3}\right. \\
& \left.\quad+\left(i A_{1}(\mathbf{x}) \partial_{3} A_{2}(\mathbf{x})-i A_{2}(\mathbf{x}) \partial_{3} A_{1}(\mathbf{x})\right) \gamma+i A_{2}(\mathbf{x}) \partial_{3} V(\mathbf{x}) \gamma \alpha_{1}-i A_{1}(\mathbf{x}) \partial_{3} V(\mathbf{x}) \gamma \alpha_{2}\right) \\
& \quad+\frac{4}{\delta^{2}}\left(\left(A_{1}^{2}(\mathbf{x})+A_{2}^{2}(\mathbf{x})\right) \alpha_{3}-A_{3}(\mathbf{x}) A_{1}(\mathbf{x}) \alpha_{1}-A_{3}(\mathbf{x}) A_{2}(\mathbf{x}) \alpha_{2}\right) \partial_{3} .
\end{aligned}
$$

Plugging (B.4), (B.5), (B.6) and (B.3) into (B.1), after some computation, we obtain (3.18). 